\title{
A Potentially Divergent and Rapid Route to Analogues of Deoxycyclitols, Pentopyranoses, 6-Deoxy-hexoses and Hexoses.
}

Christophe Audouard, John Fawcett, Gerry A. Griffith, Erwan Kérourédan, Afjal Miah, Jonathan M. Percy* and Hongli Yang.

Department of Chemistry, University of Leicester, University Road, Leicester LE1 7RH, UK.

\section{SUPPLEMENTARY INFORMATION}




\section{Supplementary Information}

Telescoped synthesis of $8 \mathrm{~b}$ as a mixture of diastereoisomers:

The following procedure was carried out in conventional glassware, either in a round bottomed flask using a slush bath (ethanol/liquid nitrogen), or in a large Schlenk tube within a metal Dewar vessel, chilled with a Huber dip cooler. The internal temperature was monitored with a thermocouple because of the exothermicity of the initial reaction.

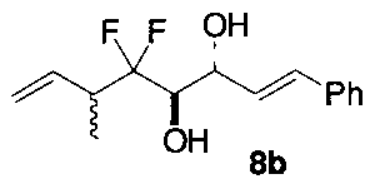

$t$-Butyllithium ( $170 \mathrm{mmol}, 100 \mathrm{~mL}$ of a $1.7 \mathrm{M}$ solution in pentane) was added by syringe pump over one hour to a solution of the ether $4 \mathrm{~b}(77 \mathrm{mmol}, 11.9 \mathrm{~g})$ in tetrahydrofuran $(300 \mathrm{~mL})$ at $-100^{\circ} \mathrm{C}$ under nitrogen and the mixture was maintained at $-100^{\circ} \mathrm{C}$ for 30 minutes. Trans-cinnamaldehyde $(70 \mathrm{mmol}, 8.7 \mathrm{~mL})$ was added dropwise over half an hour at $-100^{\circ} \mathrm{C}$ and the temperature was allowed to warm to $80^{\circ} \mathrm{C}$ over 30 minutes. The mixture was quenched at $-80^{\circ} \mathrm{C}$ with saturated aqueous ammonium chloride solution $(200 \mathrm{~mL})$ and extracted with diethyl ether $(3 \times 200 \mathrm{~mL})$. The combined organic extracts were washed with brine $(400 \mathrm{~mL})$, dried $\left(\mathrm{MgSO}_{4}\right)$ and concentrated in vacuo to afford an orange oil (ca.18.0 g).

The crude product was taken up in chloroform $(300 \mathrm{~mL})$, heated to $70^{\circ} \mathrm{C}$ and maintained at that temperature for one hour. The solution was concentrated under reduced pressure to afford the crude $\alpha$-hydroxyketone $7 \mathrm{~b}$ as an orange oil ( $\mathrm{ca} .18 .0 \mathrm{~g}$ ). The crude $\alpha$-hydroxyketone $7 \mathbf{b}$ was dissolved in diethyl ether $(200 \mathrm{~mL})$, and sodium borohydride (160 mmol, $6.0 \mathrm{~g}$ ) was added slowly at room temperature. The suspension was stirred overnight at room temperature then the reaction mixture was 
quenched carefully with concentrated hydrochloric acid $(20 \mathrm{~mL})$ and extracted with ethyl acetate $(3 \times 200 \mathrm{~mL})$. The combined organic extracts were washed with brine $(300 \mathrm{~mL})$, dried $\left(\mathrm{MgSO}_{4}\right)$ and concentrated in vacuo to leave an orange oil (17.4 g) which was purified by Kugelrohr distillation to afford diols $8 \mathrm{~b}\left(\mathrm{bp} 150^{\circ} \mathrm{C} / 0.02 \mathrm{mmHg}\right.$ ) as a mixture of diastereoisomers (ratio $1.7: 1,39.2 \mathrm{mmol}, 10.5 \mathrm{~g}, 51 \%$ over 3 steps) as white solids; $\delta_{\mathrm{H}}\left(300 \mathrm{MHz}, \mathrm{CDCl}_{3}\right) 7.29-7.12(5 \mathrm{H}, \mathrm{m}), 6.62-6-57(1 \mathrm{H}, \mathrm{m}), 6.32-6.24$ $(1 \mathrm{H}, \mathrm{m}), 5.82-5.61(1 \mathrm{H}, \mathrm{m}), 5.17-5.00(2 \mathrm{H}, \mathrm{m}), 4.53(1 \mathrm{H}, \mathrm{m}), 3.99-3.88(1 \mathrm{H}, \mathrm{m})$, 2.94-2.62 (3H, m), 1.08-1.04 (3H, m); $\delta_{\mathrm{F}}\left(\mathrm{CDCl}_{3}, 282 \mathrm{MHz}\right)-114.2\left(1 \mathrm{~F}, \mathrm{ddd},{ }^{2} J_{\mathrm{F}-\mathrm{F}}\right.$ $\left.252.1,{ }^{3} J_{\mathrm{F}-\mathrm{H}} 16.6,{ }^{3} J_{\mathrm{F}-\mathrm{H}} 14.1\right)$ major diastereoisomer, $-116.2\left(1 \mathrm{~F}, \mathrm{ddd},{ }^{2} J_{\mathrm{F}-\mathrm{F}} 251.8,{ }^{3} J_{\mathrm{F}-\mathrm{H}}\right.$ $\left.17.5,{ }^{3} J_{\mathrm{F}-\mathrm{H}} 7.3\right)$ major diastereoisomer, $-116.4\left(1 \mathrm{~F}, \mathrm{dd},{ }^{2} J_{\mathrm{F}-\mathrm{F}} 252.4,{ }^{3} J_{\mathrm{F}-\mathrm{H}} 25.9\right)$ minor

diastereoisomer, $-119.2\left(1 \mathrm{~F}\right.$, dd, $\left.{ }^{2} J_{\mathrm{F}-\mathrm{F}} 251.8,{ }^{3} J_{\mathrm{F}-\mathrm{H}} 25.1\right)$ minor diastereoisomer; $\mathrm{m} / \mathrm{z}$ (EI) $268\left(3 \%, \mathrm{M}^{+}\right), 250\left(42 \%,\left[\mathrm{M}-\mathrm{H}_{2} \mathrm{O}\right]^{+}\right)$.

The carbon spectra are complex because of extensive signal overlapping from the mixture of diastereoisomers, and are not reported numerically (the spectrum can be inspected at the end of this file).

\section{Data for pure major diastereoisomer of $8 \mathrm{~b}$ :}

A very careful flash chromatography on silica gel could allow the isolation of the pure major diastereoisomer as white flakes, $\mathrm{mp} 63-65^{\circ} \mathrm{C}$; Found: $\mathrm{C} 66.8, \mathrm{H} 6.46 \%$. Calc. for $\mathrm{C}_{15} \mathrm{H}_{18} \mathrm{~F}_{2} \mathrm{O}_{2} \mathrm{C} 67.15, \mathrm{H} 6.76 \% ; \mathrm{R}_{\mathrm{f}}$ (20\% ethyl acetate in $40-60$ petroleum ether) $0.28 ; v_{\max }(\mathrm{film}) / \mathrm{cm}^{-1}: 3325 \mathrm{~s}, 2914 \mathrm{~m}, 1724 \mathrm{w}, 1098 \mathrm{~s}, 984 \mathrm{~s}, 917 \mathrm{~s}, 690 \mathrm{~s} ; \delta_{\mathrm{H}}(300 \mathrm{MHz}$ $\left.\mathrm{CDCl}_{3}\right) 7.34-7.18(5 \mathrm{H}, \mathrm{m}), 6.65(1 \mathrm{H}, \mathrm{d}, J 15.0), 6.37(1 \mathrm{H}, \mathrm{ddt}, J 15.9, J 6.3, J 1.8)$, 5.86-5.72(1H, m), $5.14(1 \mathrm{H}, \mathrm{d}, J 15.9), 5.13(1 \mathrm{H}, \mathrm{d}, 11.7), 4.57(1 \mathrm{H}, \mathrm{br} . \mathrm{t}, J 3.0), 3.95$ (1H, br. d, $\left.{ }^{3} J_{\mathrm{H}-\mathrm{F}} 15.0\right), 2.89-2.76(1 \mathrm{H}, \mathrm{m}), 2.44(1 \mathrm{H}, \mathrm{d}, J 9.1), 2.25(1 \mathrm{H}$, br. s), 1.11 $(3 \mathrm{H}, \mathrm{d}, J 6.3) ; \delta_{\mathrm{C}}\left(75 \mathrm{MHz}, \mathrm{CDCl}_{3}\right) 136.2_{3} 135.3\left(\mathrm{t},{ }^{3} J_{\mathrm{C}-\mathrm{F}} 4.5\right), 133.0,129.0,128.1$, 
$126.9,126.7,123.5\left(\mathrm{t},{ }^{1} J_{\mathrm{C}-\mathrm{F}} 248.2\right), 118.1,73.4\left(\mathrm{dd},{ }^{2} J_{\mathrm{C}-\mathrm{F}} 24.0,{ }^{2} J_{\mathrm{C}-\mathrm{F}} 28.5\right), 71.6,42.3$

$\left(\mathrm{t},{ }^{2} J_{\mathrm{C}-\mathrm{F}} 11.2\right), 13.6\left(\mathrm{t},{ }^{3} J_{\mathrm{C}-\mathrm{F}} 4.5\right) ; \delta_{\mathrm{F}}\left(\mathrm{CDCl}_{3}, 282 \mathrm{MHz}\right)-114.2\left(1 \mathrm{~F}, \mathrm{ddd},{ }^{2} J_{\mathrm{F}-\mathrm{F}} 252.1\right.$, $\left.{ }^{3} J_{\mathrm{F}-\mathrm{H}} 16.6,{ }^{3} J_{\mathrm{F}-\mathrm{H}} 14.1\right),-116.2\left(1 \mathrm{~F}, \mathrm{ddd},{ }^{2} J_{\mathrm{F}-\mathrm{F}} 251.8,{ }^{3} J_{\mathrm{F}-\mathrm{H}} 17.5,{ }^{3} J_{\mathrm{F}-\mathrm{H}} 7.3\right) ; \mathrm{m} / z$ (EI) 268 $\left(3 \%, \mathrm{M}^{+}\right), 250\left(42 \%,\left[\mathrm{M}-\mathrm{H}_{2} \mathrm{O}\right]^{+}\right)$.

\section{Synthesis of $3 b$}

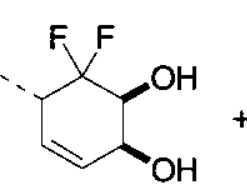

cis, trans-3b

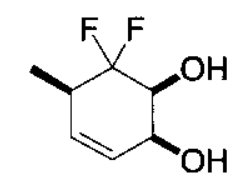

all cis-3b

Grubbs second-generation catalyst $9(0.32 \mathrm{~g}, 0.38 \mathrm{~mol}, 2 \mathrm{~mol} \%)$ was added in one portion to a solution of distilled $8 \mathbf{b}(5.10 \mathrm{~g}$ of a $1.7: 1$ mixture of diastereoisomers, $0.019 \mathrm{~mol})$ in dry, degassed $\mathrm{DCM}(760 \mathrm{~mL}, 0.025 \mathrm{M}$ in substrate $)$ and the mixture was refluxed for 24 hours at $40^{\circ} \mathrm{C}$ under a positive pressure of nitrogen. Aliquots were removed at intervals to determine if the reaction had gone to completion ( ${ }^{19} \mathrm{~F} \mathrm{NMR}$ ). The mixture was then concentrated in vacuo to afford a black oil $(5.55 \mathrm{~g})$. The mixture was purified initially by Kugelrohr distillation $\left(75-90^{\circ} \mathrm{C} / 0.04 \mathrm{mmHg}\right)$ to afford a mixture of products $(3.52 \mathrm{~g})$ contaminated by trace amounts of styrene. The products were separated using flash chromatography (Biotage, $40 \%$ ethyl acetate in $40-60$ petroleum ether) to afford cis,trans $-3 \mathrm{~b}(1.02 \mathrm{~g}, 33 \%)$, white plates, $\mathrm{mp} 88-89^{\circ} \mathrm{C}$; Found: C 51.33, H 5.85\%. Calc. for $\mathrm{C}_{7} \mathrm{H}_{10} \mathrm{~F}_{2} \mathrm{O}_{2}:$ C 51.20, H 6.09\%; $\mathrm{R}_{\mathrm{f}} 0.31(40 \%$ ethyl acetate in $40-60$ petroleum ether); $v_{\max } / \mathrm{cm}^{-1} 3432 \mathrm{~m}, 3280 \mathrm{~m}, 3020 \mathrm{w}, 2987 \mathrm{w}$, $2950 \mathrm{w}, 2890 \mathrm{w}, 1648 \mathrm{w}, 1456 \mathrm{~m}, 1427 \mathrm{~m}, 1131 \mathrm{~s}, 1067 \mathrm{~s}, 1021 \mathrm{~s}, 988 \mathrm{~s}, 916 \mathrm{~s}, 838 \mathrm{~s}, 770 \mathrm{w}$, $720 \mathrm{~s}, 655 \mathrm{w} ; \delta_{\mathrm{H}}\left(400 \mathrm{MHz}, \mathrm{CDCl}_{3}\right) 5.63-5.50(2 \mathrm{H}, \mathrm{m}), 4.42(1 \mathrm{H}$, br. s) 4.08 (1H, br. $\left.\mathrm{d},{ }^{3} J_{\mathrm{H}-\mathrm{F}} 12.0\right), 3.74(1 \mathrm{H}$, br. s), $3.12(1 \mathrm{H}, \mathrm{br} . \mathrm{s}), 2.95-2.80(1 \mathrm{H}, \mathrm{m}), 1.15(3 \mathrm{H}, \mathrm{d}, J 7.3)$; 
$\delta_{\mathrm{C}}\left(101 \mathrm{MHz}, \mathrm{CDCl}_{3}\right) 130.3\left(\mathrm{~d},{ }^{3} J_{\mathrm{C}-\mathrm{F}} 9.0\right), 126.1,122.9\left(\mathrm{dd},{ }^{1} J_{\mathrm{C}-\mathrm{F}} 249.3,244.0\right), 69.2$ (dd, $\left.{ }^{2} J_{\mathrm{C}-\mathrm{F}} 32.7,22.4\right), 68.1\left(\mathrm{~d},{ }^{3} J_{\mathrm{C}-\mathrm{F}} 5.7\right), 34.1\left(\mathrm{t},{ }^{2} J_{\mathrm{C}-\mathrm{F}} 24.2\right), 11.5\left(\mathrm{~d},{ }^{3} J_{\mathrm{C}-\mathrm{F}} 7.0\right) ; \delta_{\mathrm{F}}(376$ $\left.\mathrm{MHz}, \mathrm{CDCl}_{3}\right)-109.8\left(1 \mathrm{~F}, \mathrm{ddt},{ }^{2} J_{\mathrm{F}-\mathrm{F}} 252.5,{ }^{3} J_{\mathrm{H}-\mathrm{F}} 12.0,{ }^{4} J_{\mathrm{H}-\mathrm{F}} 7.3\right),-121.9\left(\left(1 \mathrm{~F}, \mathrm{dd},{ }^{2} J_{\mathrm{F}-\mathrm{F}}\right.\right.$ $\left.252.5,{ }^{3} J_{\mathrm{H}-\mathrm{F}} 21.4\right) ; m / z(\mathrm{EI}) 164\left(10 \%, \mathrm{M}^{+}\right), 146\left(20,\left[\mathrm{M}-\mathrm{H}_{2} \mathrm{O}\right]^{+}\right), 126\left(\left(20,\left[\mathrm{M}-\mathrm{H}_{2} \mathrm{O}\right.\right.\right.$, $\left.\mathrm{HF}]^{+}\right), 84\left(100,\left[\mathrm{CH}_{3} \mathrm{CH}=\mathrm{CHCHOH}\right]^{+}\right)$: and all cis-3b $(0.48 \mathrm{~g}, 15 \%), \mathrm{mp} 64-65^{\circ} \mathrm{C}$; Found: C 51.17, H 5.96\%. Calc. for $\mathrm{C}_{7} \mathrm{H}_{10} \mathrm{~F}_{2} \mathrm{O}_{2}: \mathrm{C} 51.20, \mathrm{H} 6.09 \%$; $\mathrm{R}_{\mathrm{f}}: 0.27(40 \%$ ethyl acetate in 40-60 petroleum ether); $v_{\max }\left(\mathrm{cm}^{-1}\right) / \mathrm{KBr} 3361 \mathrm{br}$. m, 3041w, 2978w, $2948 \mathrm{w}, 2883 \mathrm{w}, 1658 \mathrm{w}, 1463 \mathrm{~m}, 1236 \mathrm{~m}, 1108 \mathrm{~s}, 1081 \mathrm{~s}, 1017 \mathrm{~s}, 983 \mathrm{~m}, 888 \mathrm{~m}, 784 \mathrm{~m}$, 766w, 674w; $\delta_{\mathrm{H}}\left(400 \mathrm{MHz}, \mathrm{CDCl}_{3}\right)$ 5.91-5.85 (1H, m), 5.66-5.59 (1H, m) 4.39-4.30 (1H, m), 3.99-3.86 (1H, m), 3.43-3.12 (1H, br. s), 2.75-2.59 (1H, m), 2.18-1.92 (1H, m), $1.21(3 \mathrm{H}, \mathrm{d}, J 7.3) ; \delta_{\mathrm{C}}\left(101 \mathrm{MHz}, \mathrm{CDCl}_{3}\right) 132.2\left(\mathrm{~d},{ }^{3} J_{\mathrm{C}-\mathrm{F}} 8.1\right), 126.3,121.8\left(\mathrm{t},{ }^{1} J_{\mathrm{C}}\right.$ $\left.{ }_{\mathrm{F}} 246.5\right) 69.8\left(\mathrm{t},{ }^{2} J_{\mathrm{C}-\mathrm{F}} 20.8\right), 67.4\left(\mathrm{~d},{ }^{3} J_{\mathrm{C}-\mathrm{F}} 4.2\right), 38.0\left(\mathrm{t},{ }^{2} J_{\mathrm{C}-\mathrm{F}} 24.3\right), 12.1\left(\mathrm{~d},{ }^{3} J_{\mathrm{C}-\mathrm{F}} 6.5\right)$; $\delta_{\mathrm{F}}\left(376 \mathrm{MHz}, \mathrm{CDCl}_{3}\right)(-111.2)-(-112.3)(1 \mathrm{~F}, \mathrm{~m}),-128.5\left(\mathrm{ddd},{ }^{2} J_{\mathrm{F}-\mathrm{F}} 246.2,{ }^{3} J_{\mathrm{H}-\mathrm{F}} 24.4\right.$, 20.1); $m / z(E I) 164\left(10 \%, \mathrm{M}^{+}\right), 146\left(20,\left[\mathrm{M}-\mathrm{H}_{2} \mathrm{O}\right]^{+}\right), 126\left(\left(20,\left[\mathrm{M}-\mathrm{H}_{2} \mathrm{O}, \mathrm{HF}\right]^{+}\right), 84\right.$ $\left(100,\left[\mathrm{CH}_{3} \mathrm{CH}=\mathrm{CHCHOH}\right]^{+}\right)$and a mixed fraction consisting of the two stereoisomers $(0.51 \mathrm{~g}, 16 \%)$.

Crystals of both stereoisomers for $\mathrm{X}$-ray crystallographic analysis were grown from dichloromethane by slow evaporation. 


\section{Preparation of 8c (mixture of diastereoisomers)}

Dehydrofluorination/metallation and addition to acrolein; preparation of $6 \mathrm{c}$<smiles>C=CC(O)C(OCC=CCc1ccccc1)=C(F)F</smiles>

$n$-Butyllithium ( $5 \mathrm{mmol}, 2 \mathrm{~mL}$ of a $2.5 \mathrm{M}$ solution in hexanes) was added dropwise over 20 minutes to a stirred solution of $4 \mathrm{c}(0.60 \mathrm{~g}, 2.3 \mathrm{mmol})$ in dry THF (12 mL) at $78^{\circ} \mathrm{C}$ under nitrogen. After 20 minutes, acrolein $(0.28 \mathrm{~g}, 5 \mathrm{mmol})$ was added in one portion. The temperature was then allowed to rise from $-78{ }^{\circ} \mathrm{C}$ to $-40{ }^{\circ} \mathrm{C}$ over 1 hour. The reaction was quenched with ammonium chloride $(10 \mathrm{~mL}$ of a saturated aqueous solution) and extracted with diethyl ether $(3 \times 10 \mathrm{~mL})$. The combined organic extracts were washed with water $(20 \mathrm{~mL})$ then brine $(20 \mathrm{~mL})$, dried $\left(\mathrm{MgSO}_{4}\right)$ and concentrated under reduced pressure gave afford an orange oil $(0.6 \mathrm{~g}) .{ }^{19} \mathrm{~F}$ NMR showed the complete consumption of $\mathbf{4 c}$ and the appearance of a new product, which was not characterised or further or purified. The data are consistent with the formation of $6 \mathrm{c}$ : $\delta_{\mathrm{F}}\left(282 \mathrm{MHz} ; \mathrm{CDCl}_{3}\right)-99.1\left(\mathrm{~d},{ }^{2} J_{\mathrm{F}-\mathrm{F}} 68.7\right),-110.6\left(\mathrm{dd},{ }^{2} J_{\mathrm{F}-\mathrm{F}} 68.7,{ }^{4} J_{\mathrm{F}-\mathrm{H}} 2.9\right)$.

\section{Claisen rearrangement to $7 c$}

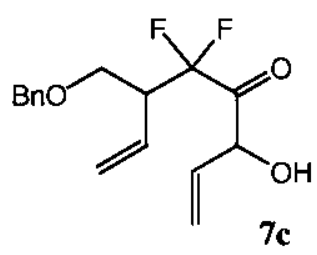

A solution of crude $6 \mathrm{c}(0.60 \mathrm{~g})$ in dry chloroform $(10 \mathrm{~mL})$ was heated at $85^{\circ} \mathrm{C}$ for 3hours in an Ace tube. The tube was cooled to room temperature, opened carefully and the contents were concentrated under reduced pressure to afford a yellow oil $(0.59$ 
g). ${ }^{19} \mathrm{~F}$ NMR showed the complete consumption of $\mathbf{6 c}$ and the appearance of a new product, which was not characterised or further or purified. The data are consistent with the formation of $7 \mathrm{c}: \delta_{\mathrm{F}}\left(282 \mathrm{MHz} ; \mathrm{CDCl}_{3}\right)-109.4\left(\mathrm{dd},{ }^{2} J_{\mathrm{F}-\mathrm{F}} 269.2,{ }^{3} J_{\mathrm{F}-\mathrm{H}} 13.3\right)$, $111.8\left(\mathrm{dd},{ }^{2} J_{\mathrm{F}-\mathrm{F}} 269.1,{ }^{3} J_{\mathrm{F}-\mathrm{H}} 16.1\right)$.

\section{Reduction to $8 \mathrm{c}$}

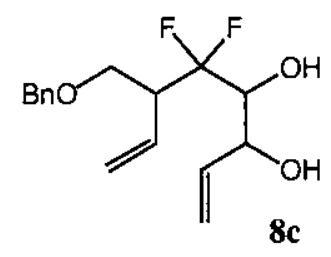

Sodium borohydride $(0.30 \mathrm{~g}, 7.96 \mathrm{mmol})$ was added to a solution of crude $\alpha$ hydroxyketone $7 \mathrm{c}(0.59 \mathrm{~g}, 1.99 \mathrm{mmol})$ in ethanol $(16 \mathrm{ml})$ at room temperature. The mixture was stirred at room temperature overnight, then quenched with ammonium chloride (15 mL of a saturated aqueous solution). The mixture was extracted with diethyl ether ( $3 \times 15 \mathrm{~mL})$ and the combined organic extracts were washed with brine $(15 \mathrm{~mL})$ and dried $\left(\mathrm{MgSO}_{4}\right)$. The solvent was removed under vacuum to afford a viscous oil which was purified by flash column chromatography (Biotage, silica gel, $40 \%$ ethyl acetate/petroleum ether) to afford $8 \mathrm{c}(0.208 \mathrm{~g}, 35 \%)$ as a mixture of diastereoisomers (3.6:1) which could be taken on through RCM and then separated: mp 49-52 ${ }^{\circ} \mathrm{C}$; Found: C 64.72, H 6.86\%. Calc. for $\mathrm{C}_{16} \mathrm{H}_{20} \mathrm{~F}_{2} \mathrm{O}_{3}: \mathrm{C} 64.42, \mathrm{H} 6.76 \%$ $\delta_{\mathrm{H}}\left(300 \mathrm{MHz}, \mathrm{CDCl}_{3}\right) 7.36-7.15(5 \mathrm{H}, \mathrm{m}), 6.01-5.60(1 \mathrm{H}, \mathrm{m}), 5.39-5.09(2 \mathrm{H}, \mathrm{m}), 4.48$ $(2 \mathrm{H}, \mathrm{s}$, minor diastereoisomer $), 4.41(2 \mathrm{H}, \mathrm{s}$, major diastereoisomer $), 4.48-4.32(1 \mathrm{H}$, br. s), 3.92-3.50 (3H, m), 3.23-2.96 $(2 \mathrm{H}, \mathrm{m}), 2.55(1 \mathrm{H}$, br. s, major diastereoisomer), $2.40\left(1 \mathrm{H}\right.$, br. s, minor diastereoisomer); $m / z(\mathrm{EI}) 298\left(23 \%, \mathrm{M}^{+}\right), 107\left(35,\left[\mathrm{C}_{7} \mathrm{H}_{7} \mathrm{O}\right]^{+}\right)$, $91\left(100,\left[\mathrm{C}_{7} \mathrm{H}_{7}\right]^{+}\right)$. The ${ }^{13} \mathrm{C}$ NMR spectrum was overlapped and no further 
characterisation was obtained. To support the structural assignment of this mixture, the major diol was purified rigorously by very careful chromatography (as above): $\mathrm{mp}$ 72-75 ${ }^{\circ} \mathrm{C}$; Found: C 64.46, H 6.87\%. Calc. for $\mathrm{C}_{16} \mathrm{H}_{20} \mathrm{~F}_{2} \mathrm{O}_{3}: \mathrm{C} 64.42, \mathrm{H} 6.76 \%$. $\mathrm{R}_{\mathrm{f}} 0.63$ ( $40 \%$ ethyl acetate / petroleum ether); $v_{\max }(\mathrm{KBr}) / \mathrm{cm}^{-1}: 3346,2914,2880,1453,1362$, $1205,1087,1005,922,755,695 ; \delta_{\mathrm{H}}\left(300 \mathrm{MHz} ; \mathrm{CDCl}_{3}\right) 7.30-7.16(5 \mathrm{H}, \mathrm{m}), 5.93(1 \mathrm{H}$, ddddd, $\left.J 17.2,10.5,5.6,{ }^{4} J 2.1,1.0\right), 5.71(1 \mathrm{H}$, ddd, $J 17.1,10.5,1.5), 5.21(1 \mathrm{H}, \mathrm{dt}, J$ $17.1,1.5), 5.18(1 \mathrm{H}$, br. d, app. $J 17.2), 5.15(2 \mathrm{H}, \mathrm{dt}, J 10.5,1.5), 4.41(2 \mathrm{H}, \mathrm{s}), 3.92-$ $3.78(1 \mathrm{H}, \mathrm{m}), 3.69\left(1 \mathrm{H}, \mathrm{dd},{ }^{2} J 9.8, J 5.8\right), 3.50\left(1 \mathrm{H}, \mathrm{ddd},{ }^{2} J 9.8, J 6.6,{ }^{4} J 1.1\right), 2.55$ (1H, br. s); $\delta_{\mathrm{C}}\left(75 \mathrm{MHz} ; \mathrm{CDCl}_{3}\right) 137.5,136.0\left(\mathrm{~d},{ }^{4} J_{\mathrm{C}-\mathrm{F}} 2.4\right), 132.0\left(\mathrm{dd},{ }^{3} J_{\mathrm{C}-\mathrm{F}} 3.6,3.6\right)$, $128.5,127.9,127.8,123.1\left(\mathrm{dd},{ }^{1} J_{\mathrm{C}-\mathrm{F}} 249.0,247.7\right), 120.9,117.3,73.42\left(\mathrm{dd},{ }^{2} J_{\mathrm{C}-\mathrm{F}} 22.7\right.$, 22.7), 73.4, $71.3\left(\mathrm{~d},{ }^{3} J_{\mathrm{C}-\mathrm{F}} 2.4\right), 67.9\left(\mathrm{t},{ }^{3} J_{\mathrm{C}-\mathrm{F}} 4.8\right), 48.9\left(\mathrm{t},{ }^{2} J_{\mathrm{C}-\mathrm{F}} 21.5\right) ; \delta_{\mathrm{F}}(282 \mathrm{MHz}$; $\left.\mathrm{CDCl}_{3}\right)-112.3\left(1 \mathrm{~F}, \mathrm{ddd},{ }^{2} J_{\mathrm{F}-\mathrm{F}} 254.6,{ }^{3} J_{\mathrm{F}-\mathrm{H}} 20.0,3.8\right),-116.3\left(1 \mathrm{~F}, \mathrm{ddd},{ }^{2} J_{\mathrm{F}-\mathrm{F}} 254.6,{ }^{3} J_{\mathrm{F}-\mathrm{H}}\right.$ $21.1,10.4) ; m / z(\mathrm{EI}): 298\left(21 \%, \mathrm{M}^{+}\right), 280\left(8 \%, \mathrm{M}^{+}-\mathrm{H}_{2} \mathrm{O}\right), 91\left(100 \%, \mathrm{C}_{7} \mathrm{H}_{7}^{+}\right)$.

\section{Preparation of 15}

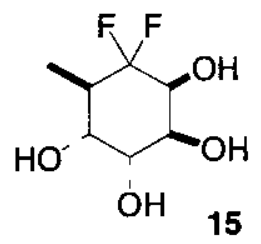

An solution of NMO $(0.14 \mathrm{~g}, 1.2 \mathrm{mmol})$ in water $(0.2 \mathrm{ml})$ was added at room temperature to a stirred solution of all $c i s-3 \mathrm{~b}(0.10 \mathrm{~g}, 0.6 \mathrm{mmol})$ in acetone $(0.6 \mathrm{~mL})$ and ${ }^{\mathrm{t}} \mathrm{BuOH}(0.6 \mathrm{~mL})$. After stirring for 15 minutes, the mixture was cooled to $0^{\circ} \mathrm{C}$, and osmium tetroxide $(0.12 \mathrm{ml}$ of a $2.5 \%$ solution in tert $-\mathrm{BuOH}, 12.2 \mu \mathrm{mol})$ was added dropwise. The mixture was allowed to warm to room temperature and stirred for 7days. Sodium sulphite was added to quench the reaction and the mixture was stirred for 15 minutes. The mixture was then filtered through a plug of silica gel, which was 
washed repeatedly with ethyl acetate $(5 \times 10 \mathrm{~mL})$ and the filtrate was concentrated under reduce pressure. The crude product was purified by flash chromatography ( $5 \%$ methanol in dichloromethane) to yield $15(0.08 \mathrm{~g}, 79 \%)$ as a powder mp $122-124^{\circ} \mathrm{C}$; Found: $\mathrm{C} 42.48$, H 5.96\%. Calc. for $\mathrm{C}_{7} \mathrm{H}_{12} \mathrm{~F}_{2} \mathrm{O}_{4}: \mathrm{C} 42.43, \mathrm{H} 6.10 \%$. $\mathrm{R}_{\mathrm{f}}=0.07(10 \%$ methanol in dichloromethane); $v_{\max } / \mathrm{cm}^{-1}(\mathrm{KBr}) 3560 \mathrm{w}, 3314 \mathrm{br} . \mathrm{m}, 2921 \mathrm{w}, 1411 \mathrm{~m}$, $1380 \mathrm{w}, 1304 \mathrm{~m}, 1225 \mathrm{w}, 1113 \mathrm{~m}, 1074 \mathrm{~s}, 1022 \mathrm{~s}, 995 \mathrm{~s}, 960 \mathrm{~m}, 896 \mathrm{~m}, 809 \mathrm{~m}, 772 \mathrm{~m}$, $707 \mathrm{~m} ; \delta_{\mathrm{H}}\left(400 \mathrm{MHz}, \mathrm{CD}_{3} \mathrm{OD}\right) 4.03-3.89(3 \mathrm{H}, \mathrm{m}), 3.66\left(1 \mathrm{H}, \mathrm{dd},{ }^{3} J_{\mathrm{H}-\mathrm{F}} 11.0, J 2.7\right)$, $2.22 *\left(1 \mathrm{H}, \mathrm{dddq},{ }^{3} J_{\mathrm{H}-\mathrm{F}} 24.8,7.9, J 13.0,6.8\right), 1.16(3 \mathrm{H}, \mathrm{d}, J 6.8) ; \delta_{\mathrm{C}}(100 \mathrm{MHz}$, $\left.\mathrm{CD}_{3} \mathrm{OD}\right) 122.1$ (dd, $\left.{ }^{1} J_{\mathrm{C}-\mathrm{F}} 250.8,244.4\right), 72.2,71.9\left(\mathrm{~d},{ }^{3} J_{\mathrm{C}-\mathrm{F}} 8.2\right), 69.6\left(\mathrm{~d},{ }^{3} J_{\mathrm{C}-\mathrm{F}} 9.1\right.$ ), $68.3\left(\mathrm{dd},{ }^{2} J_{\mathrm{C}-\mathrm{F}} 21.0,20.2\right), 37.9\left(\mathrm{t},{ }^{2} J_{\mathrm{C}-\mathrm{F}} 20.8\right), 7.1 ; \delta_{\mathrm{F}}\left(376 \mathrm{MHz}, \mathrm{CD}_{3} \mathrm{OD}, 218 \mathrm{~K}\right)-$ $105.7\left(1 \mathrm{~F}, \mathrm{~d},{ }^{2} J_{\mathrm{F}-\mathrm{F}} 243.8\right),-126.7\left(1 \mathrm{~F}, \mathrm{dt},{ }^{2} J_{\mathrm{F}-\mathrm{F}} 243.8,{ }^{3} J_{\mathrm{H}-\mathrm{F}} 24.8\right) ; \mathrm{m} / \mathrm{z} \quad$ (CI): 216 $\left(100 \%,\left[\mathrm{M}+\mathrm{NH}_{4}\right]^{+}\right)$.

The complex signal at $2.22 \mathrm{ppm}$ was simulated using gNMR 5.0 (Adept Scientific) and estimated values for the trans-diaxial coupling and one of the H-F couplings, which cannot be measured in the ${ }^{19} \mathrm{~F}$ NMR spectrum. 


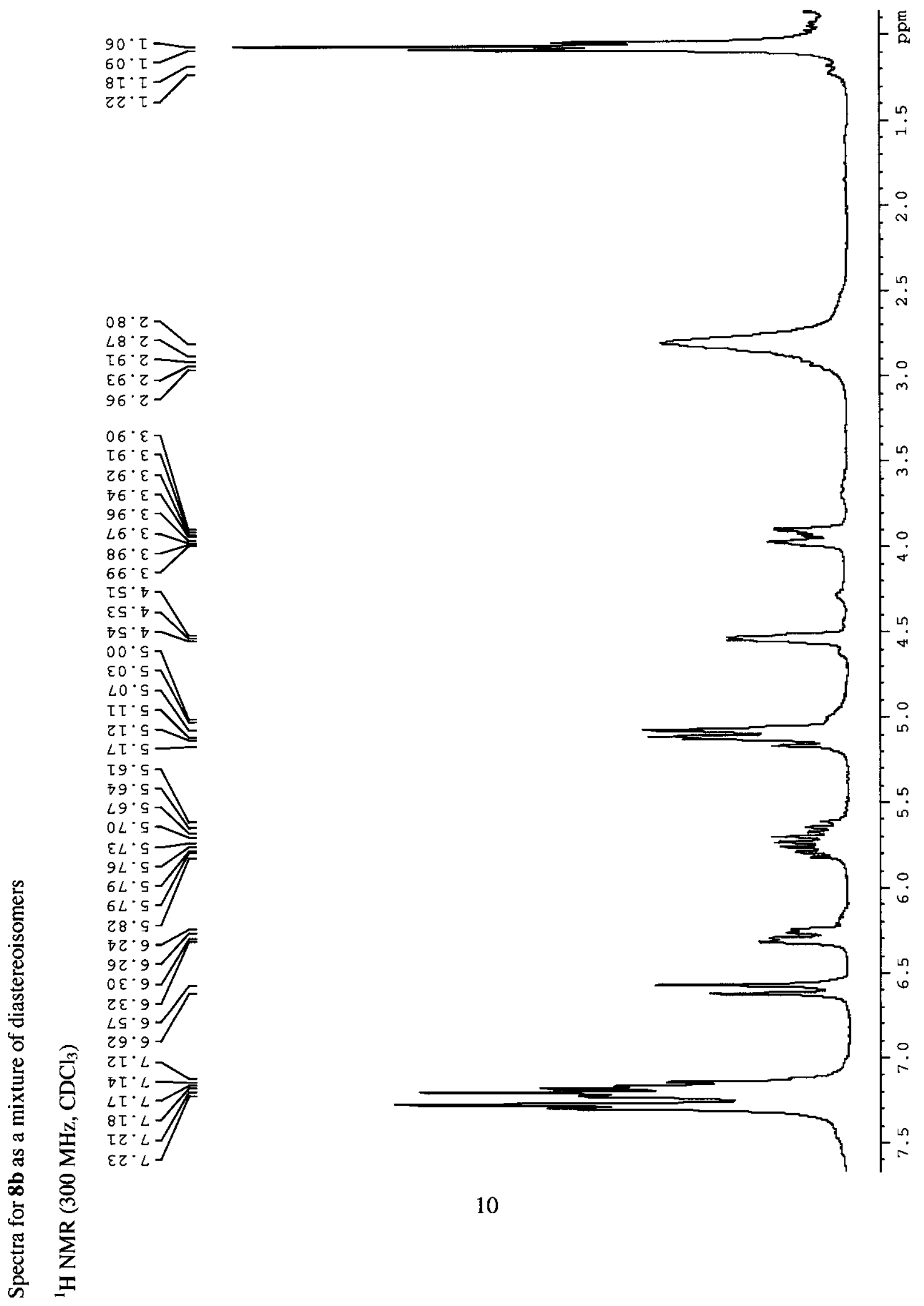




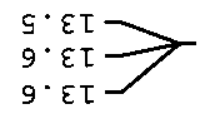

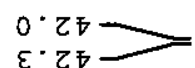
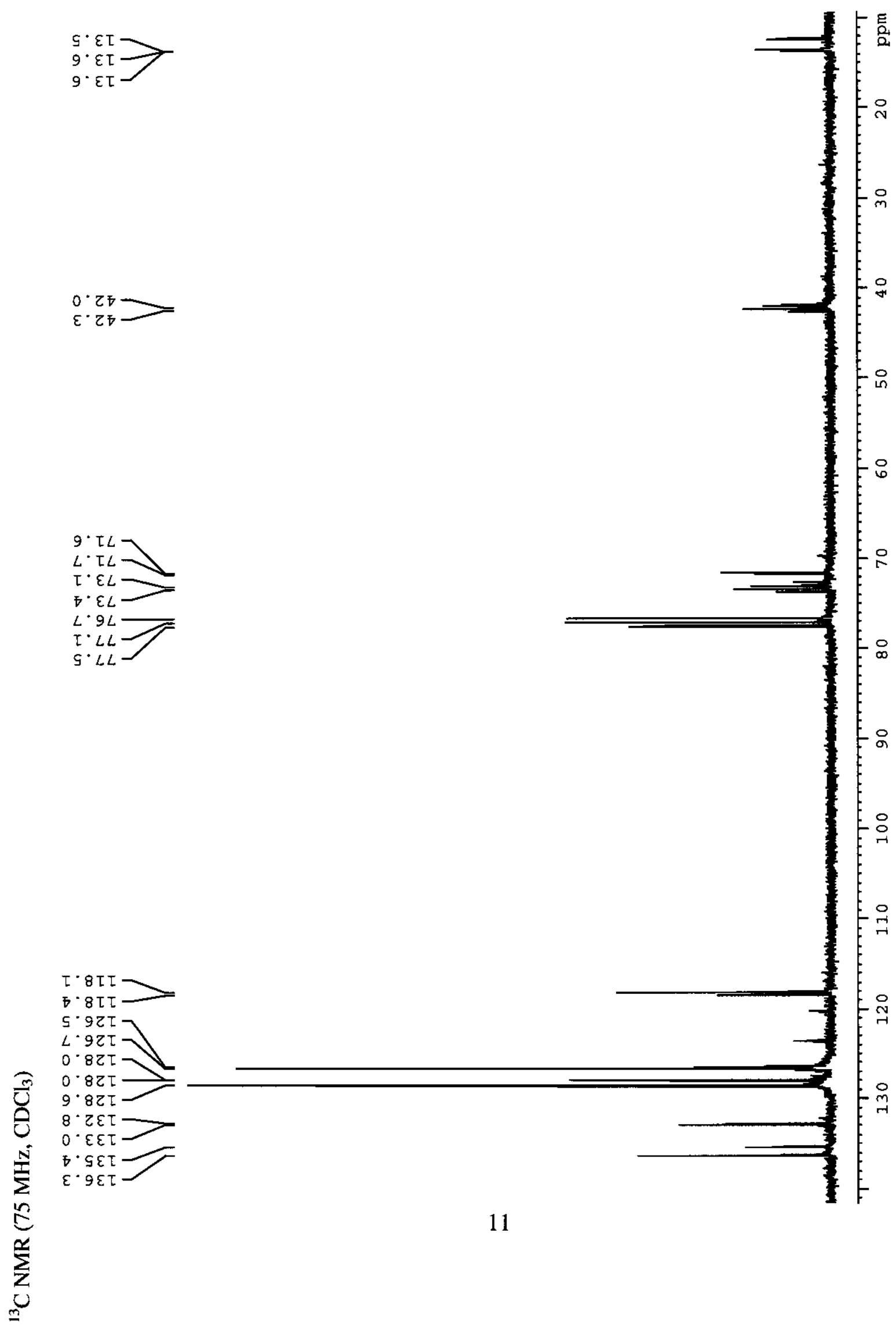

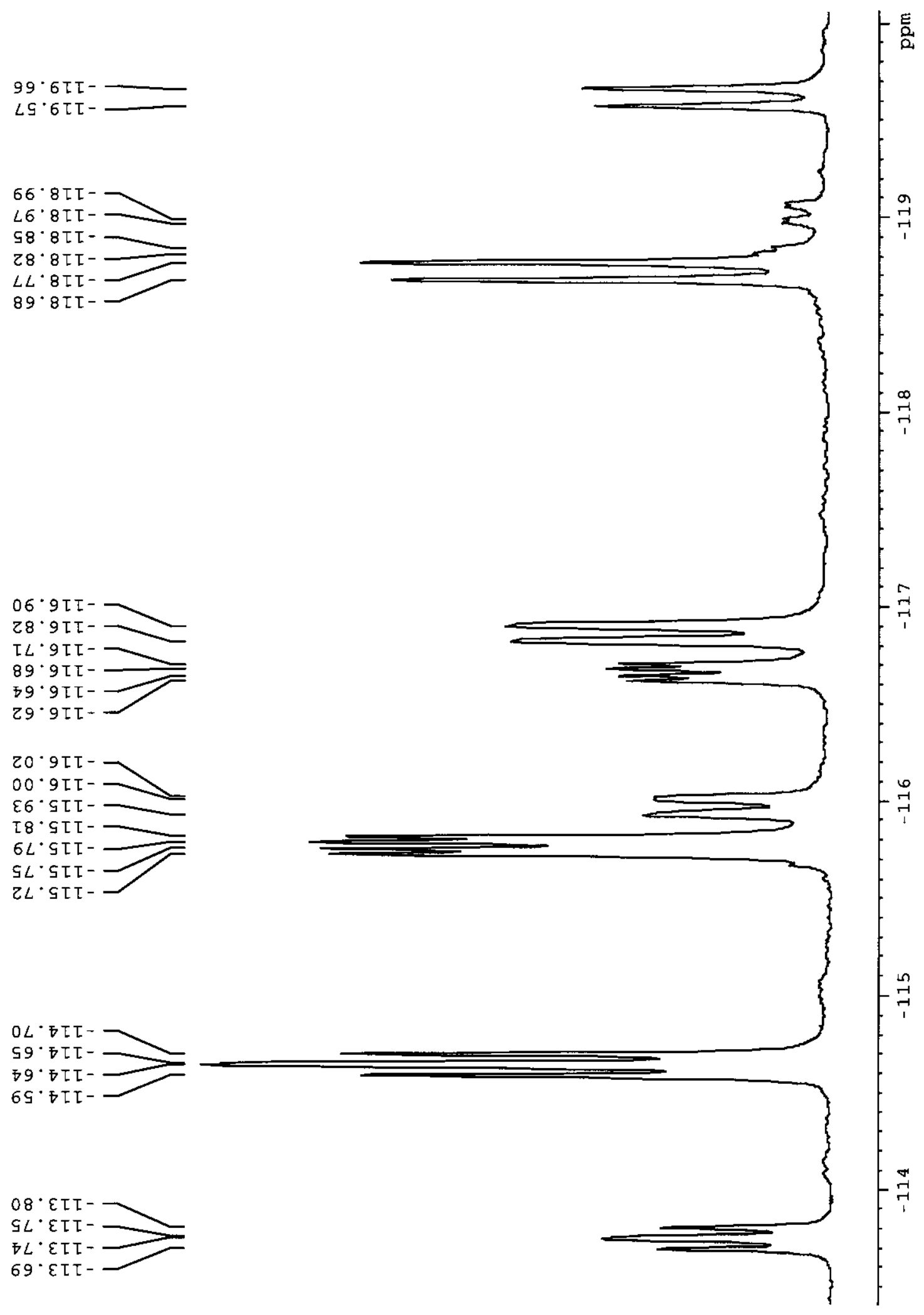


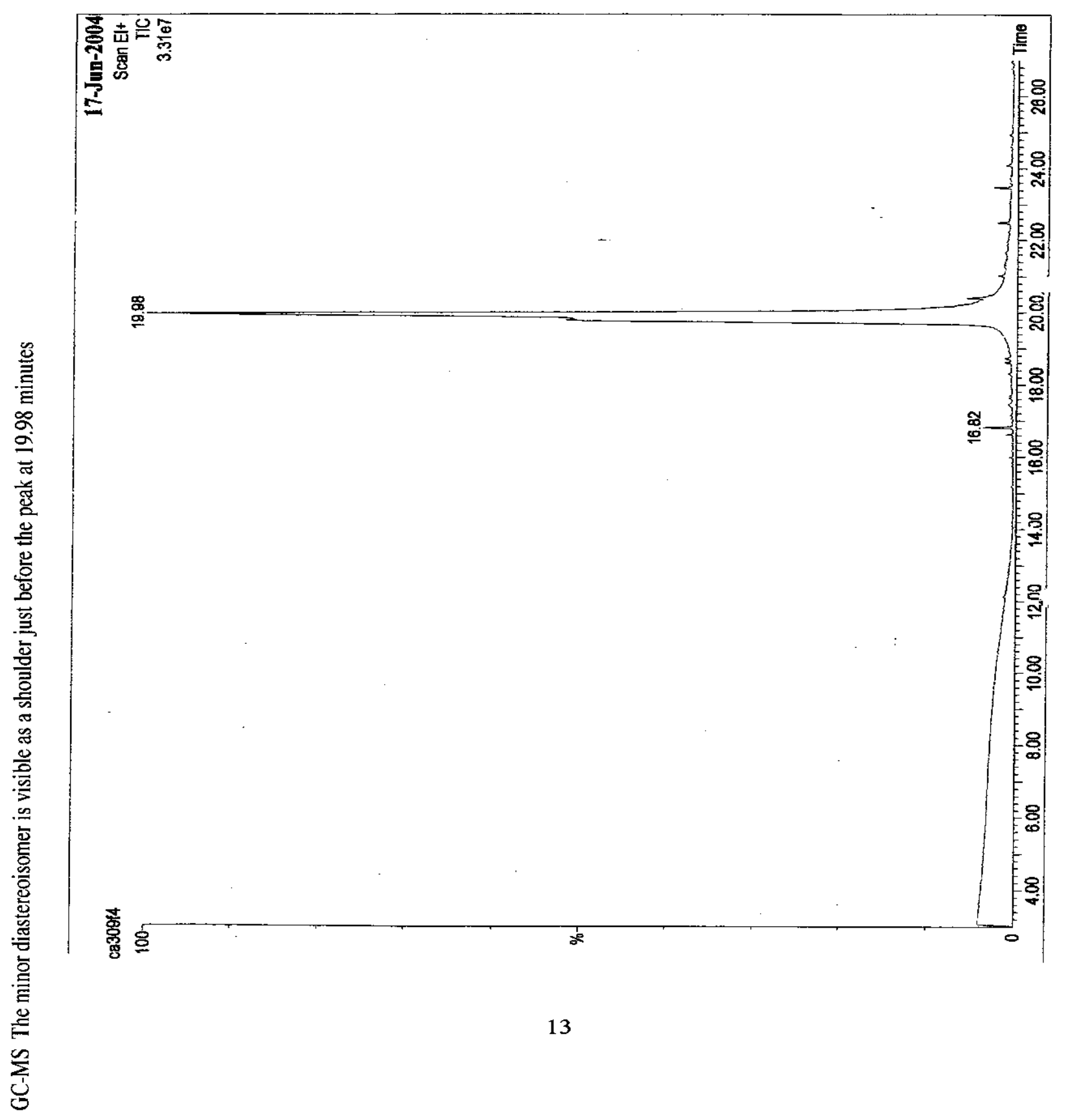




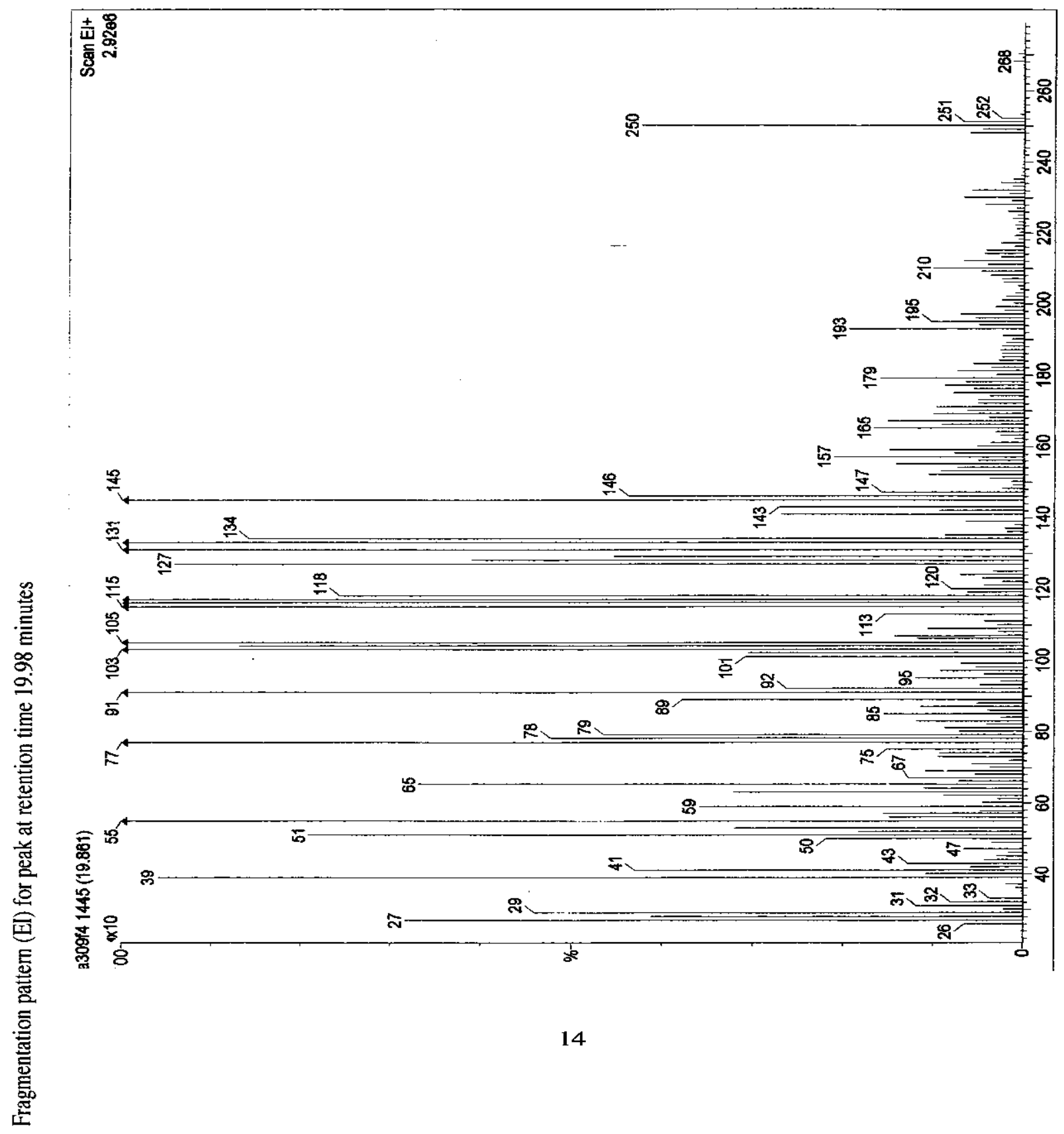



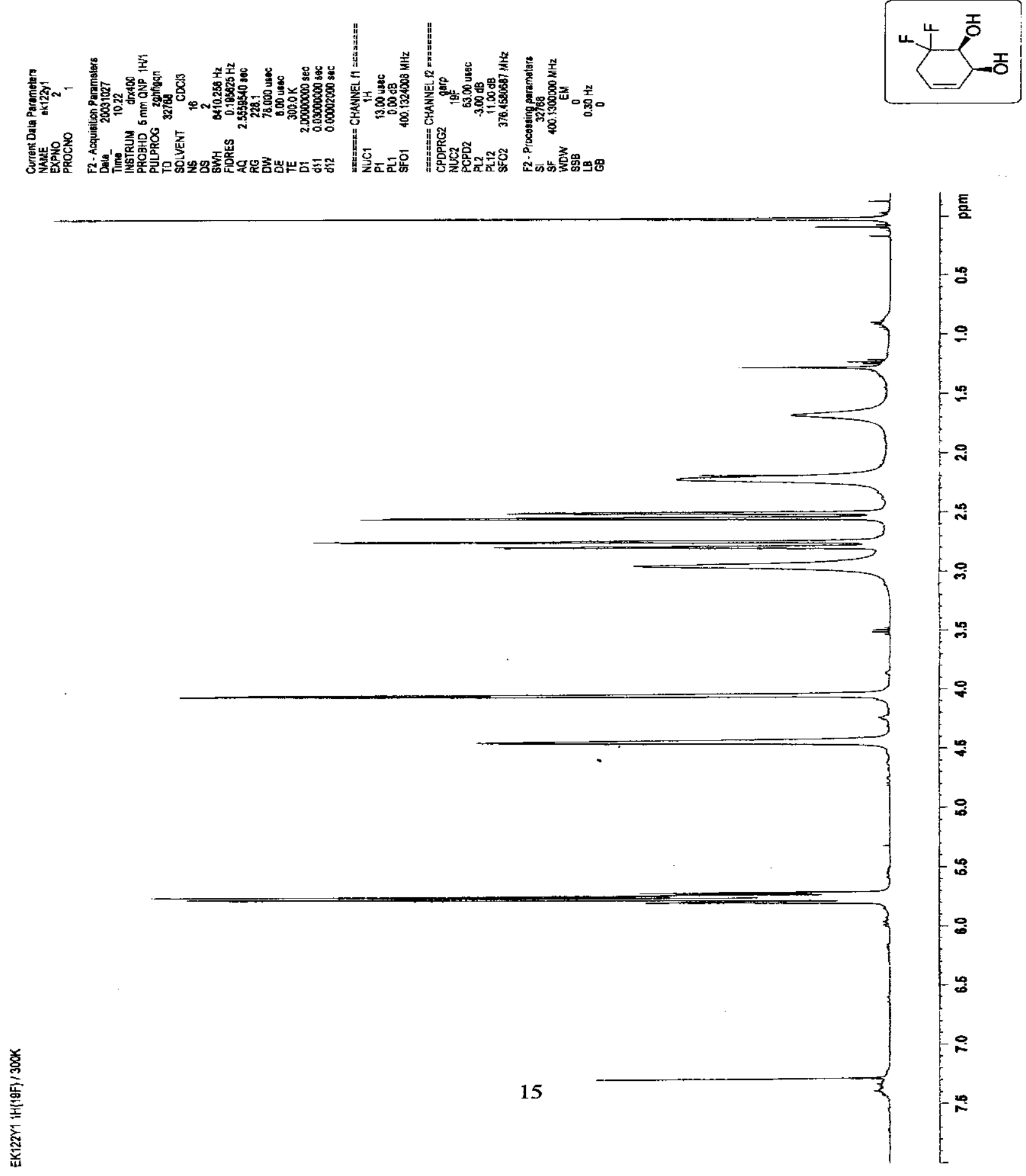

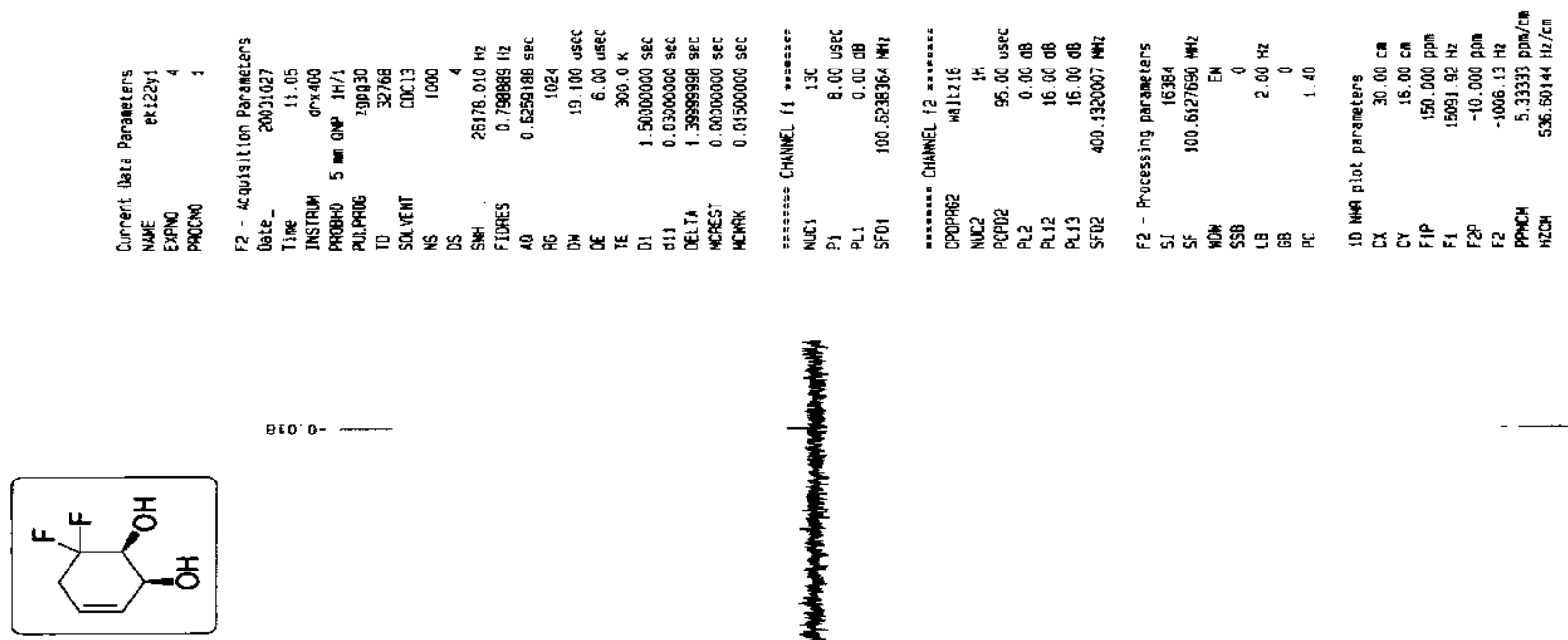

860.

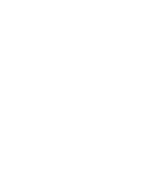

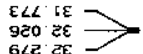

$521<9$

622.69
086.69

8rg.69

$560^{\circ} .92 \longrightarrow$

oEE $\angle L \supset$

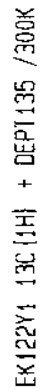

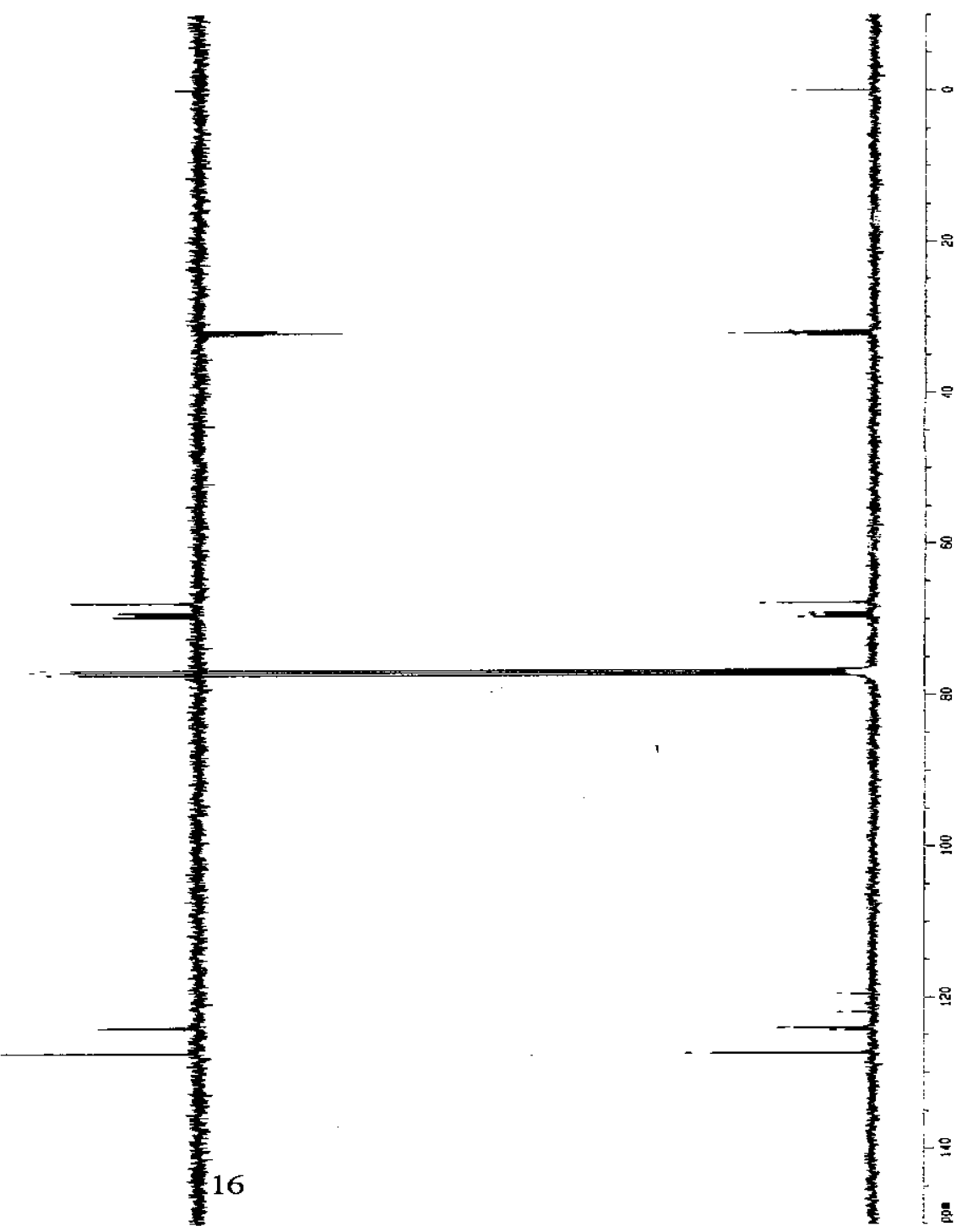




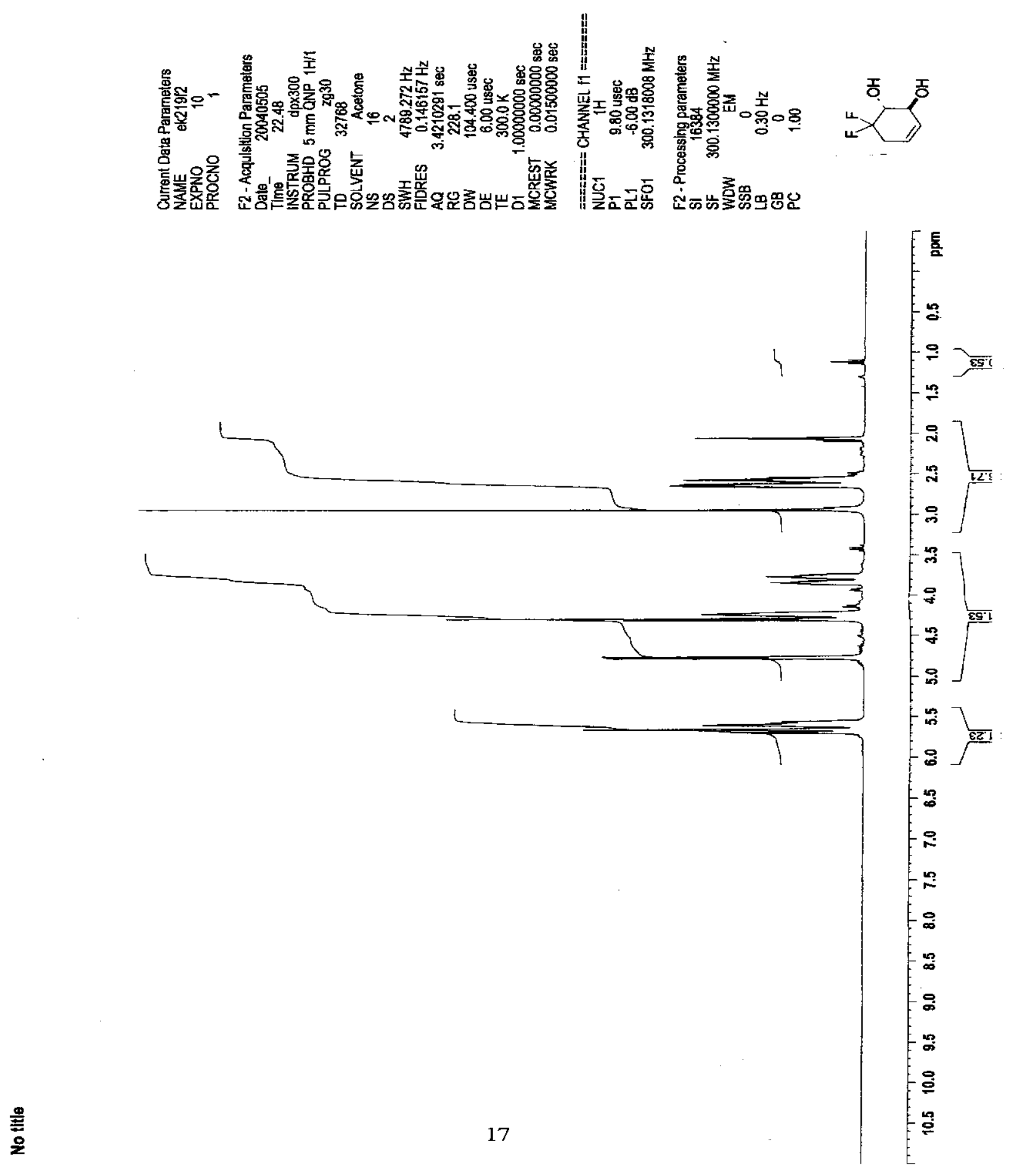





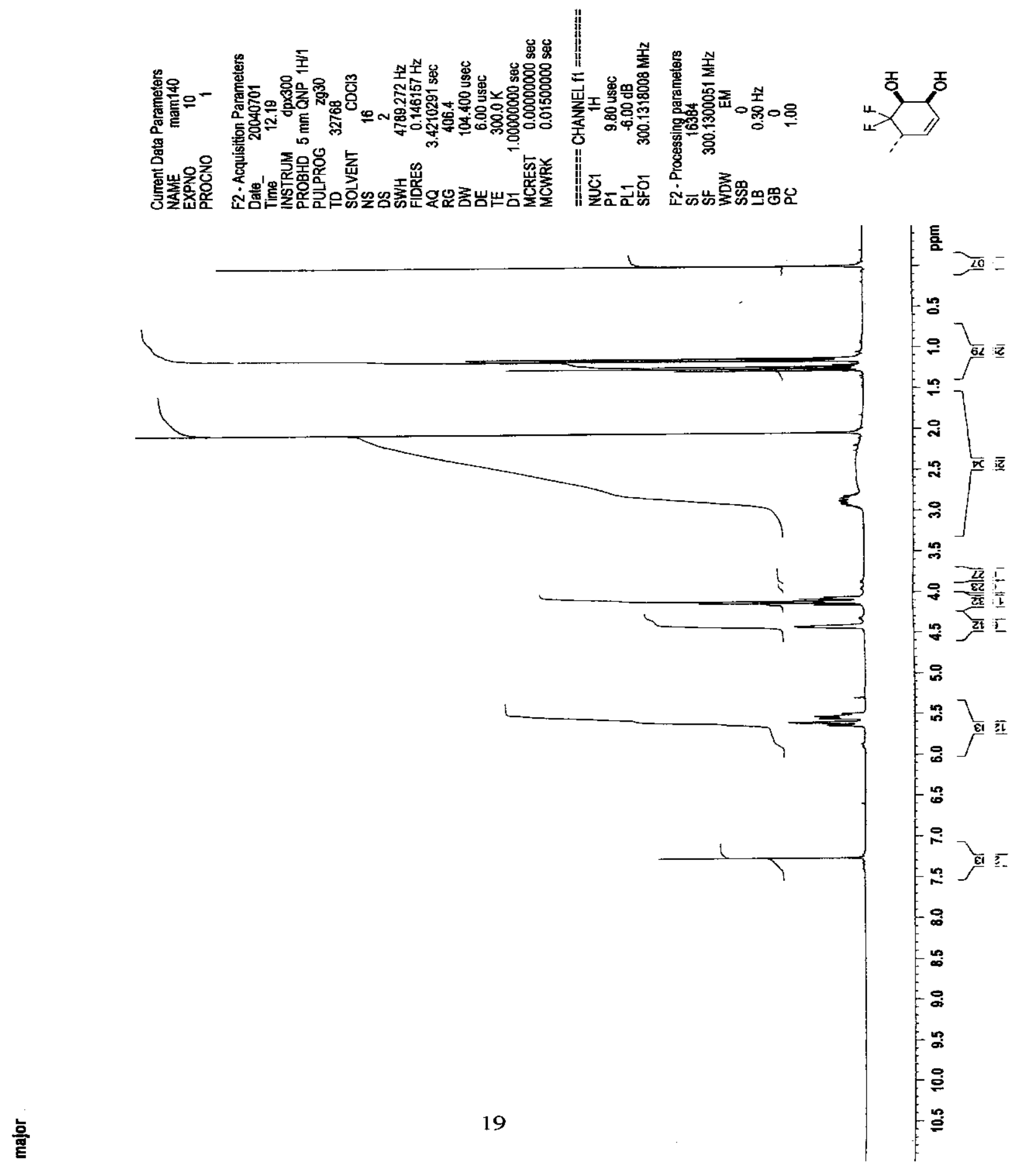




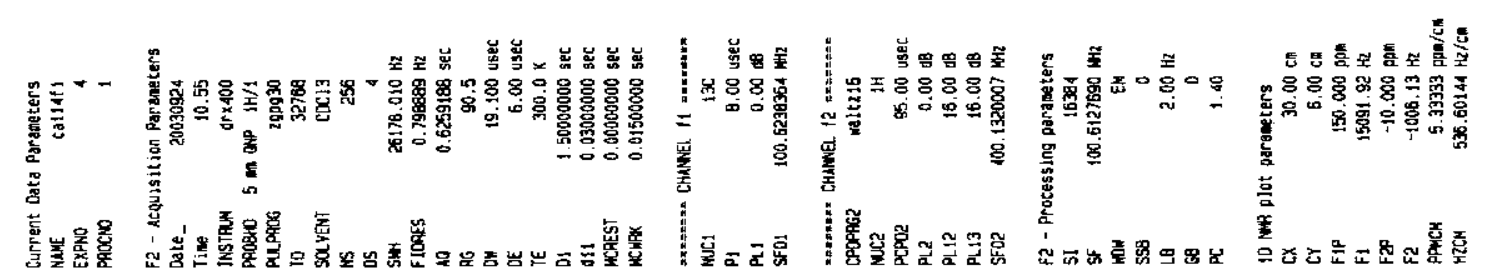

?

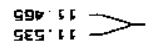

$\angle E g \cdot E E$

GOE'
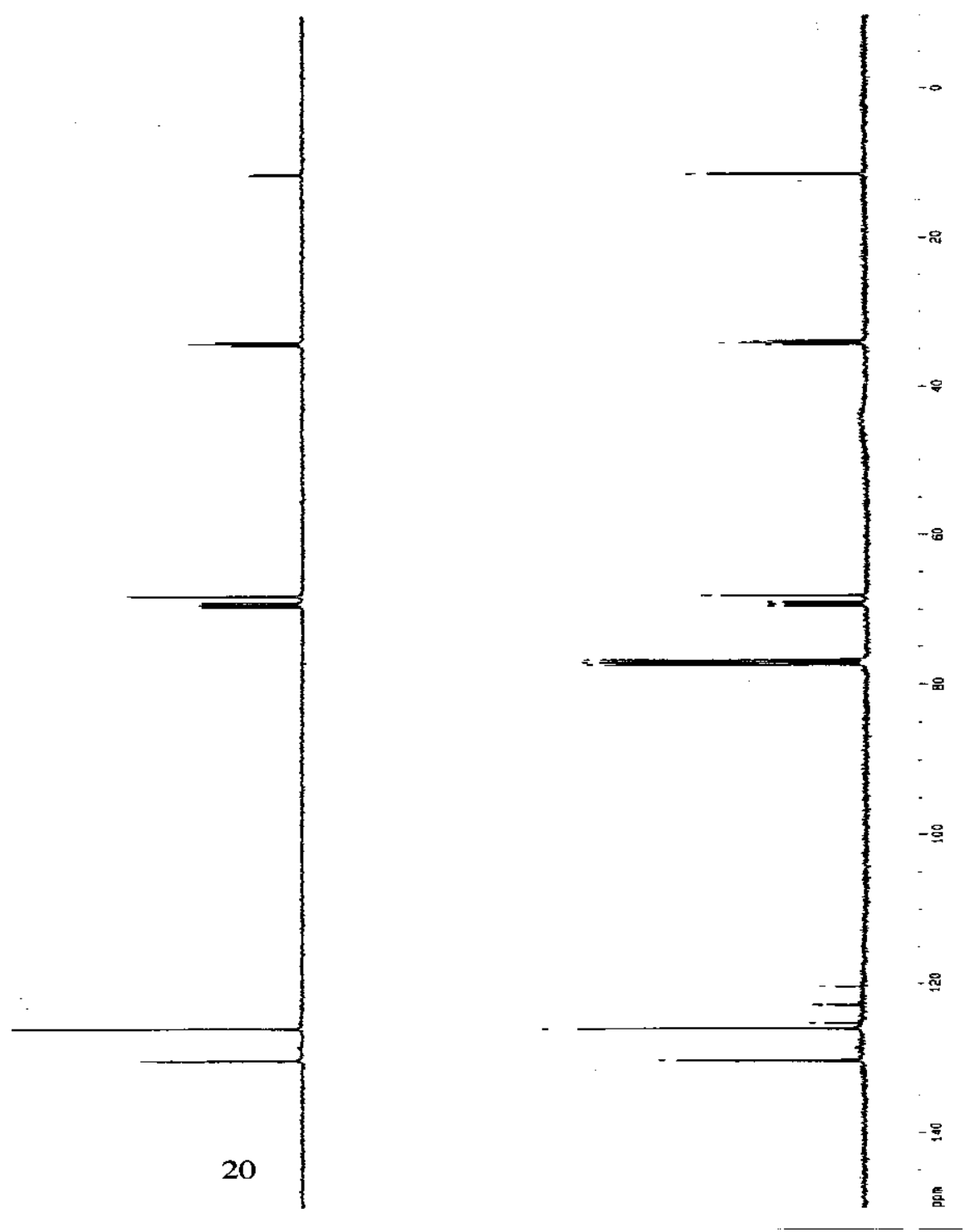


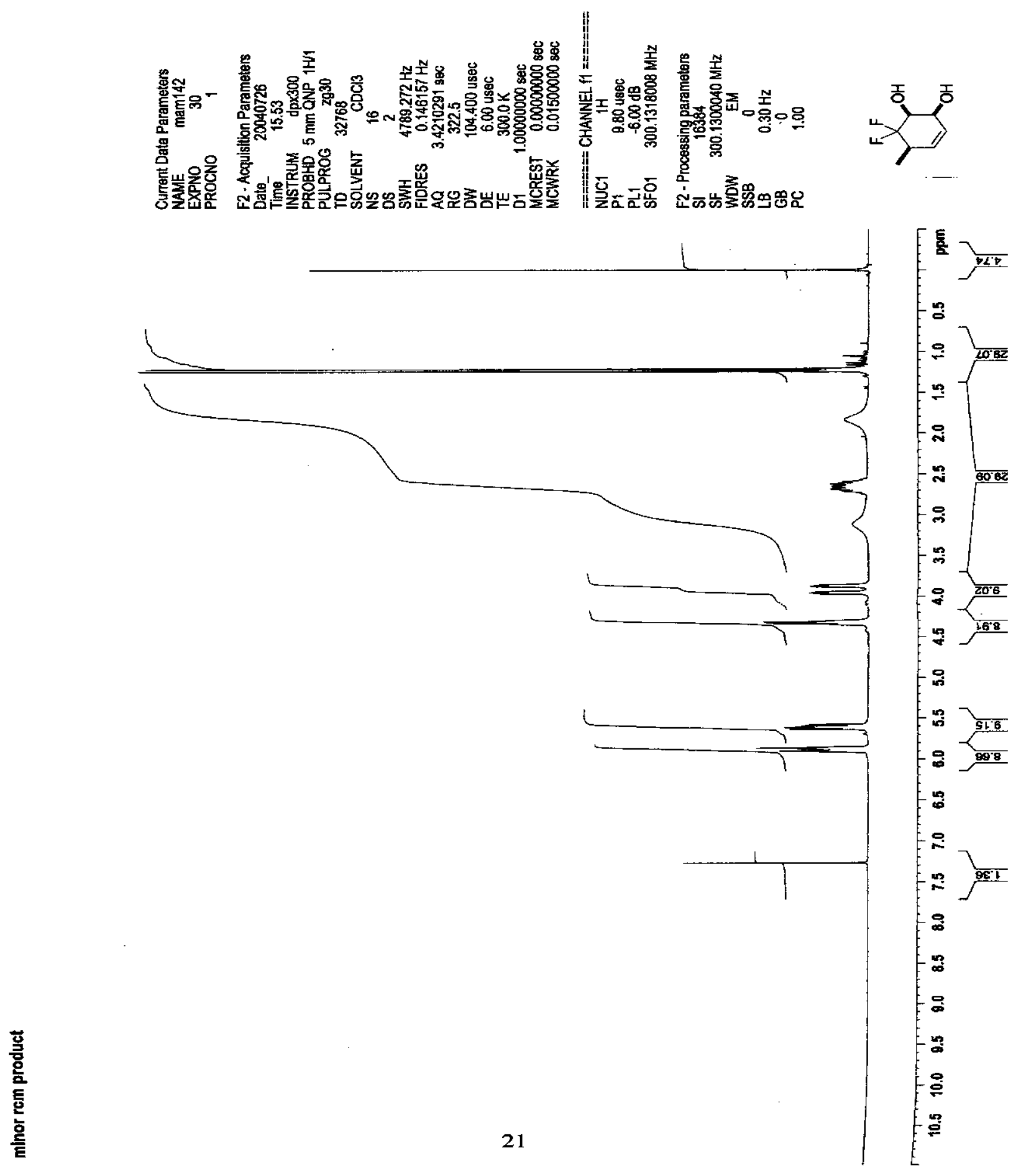




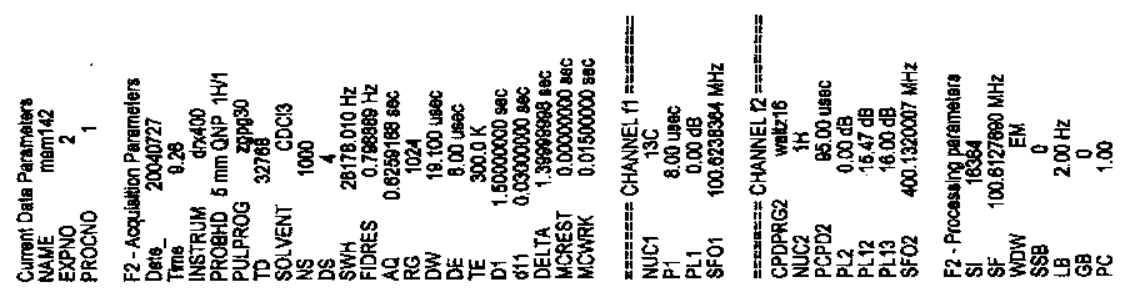

告

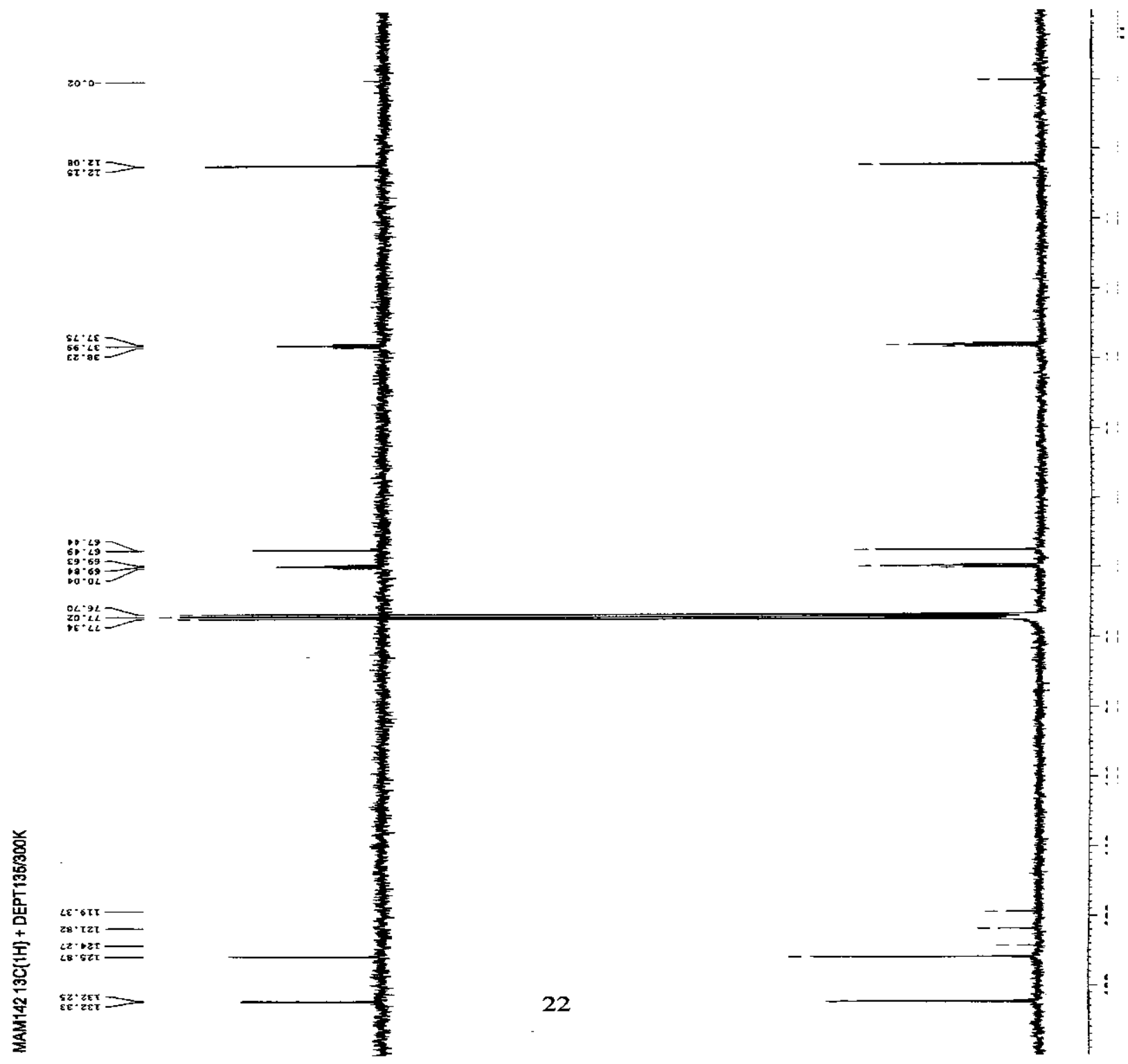




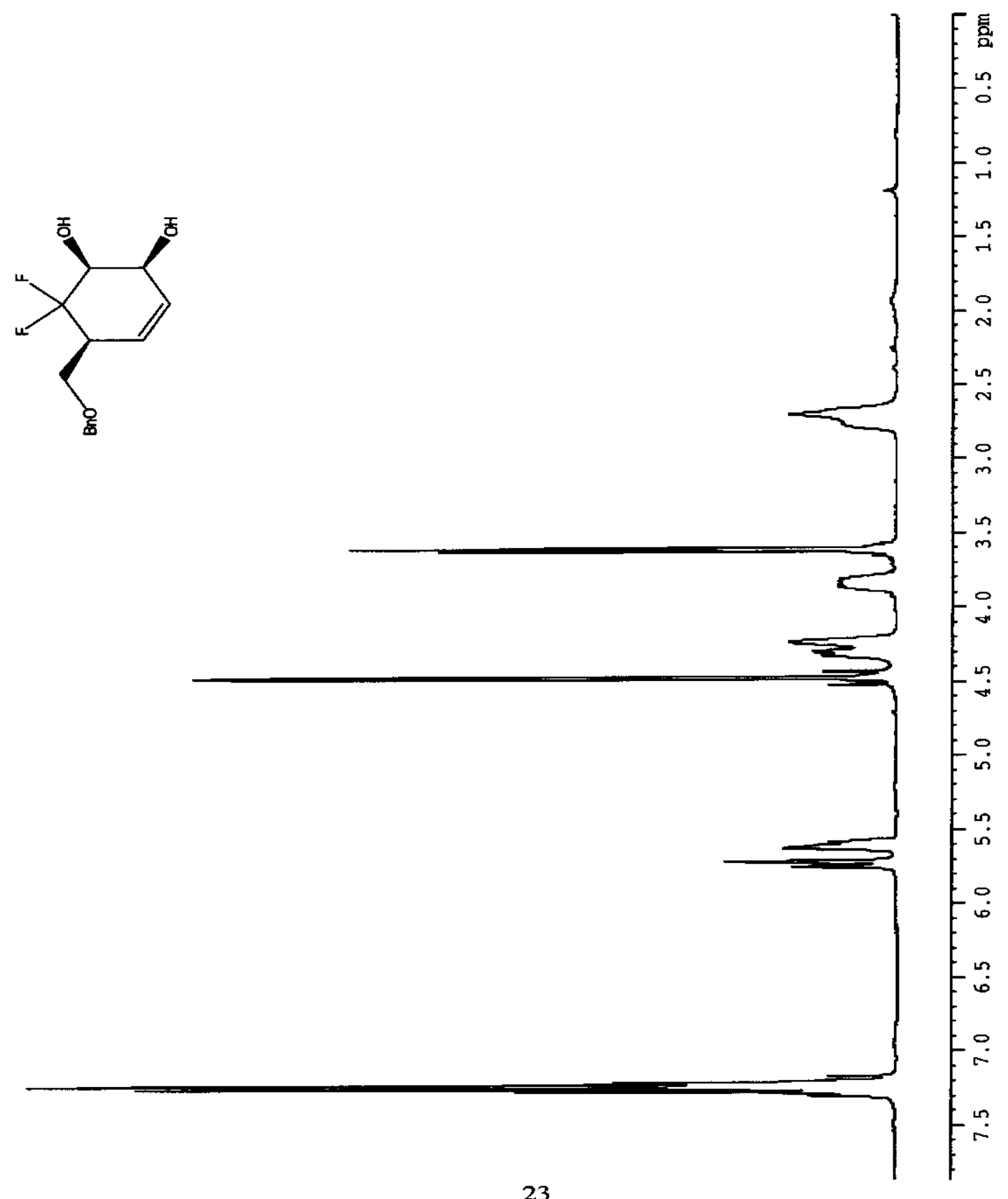



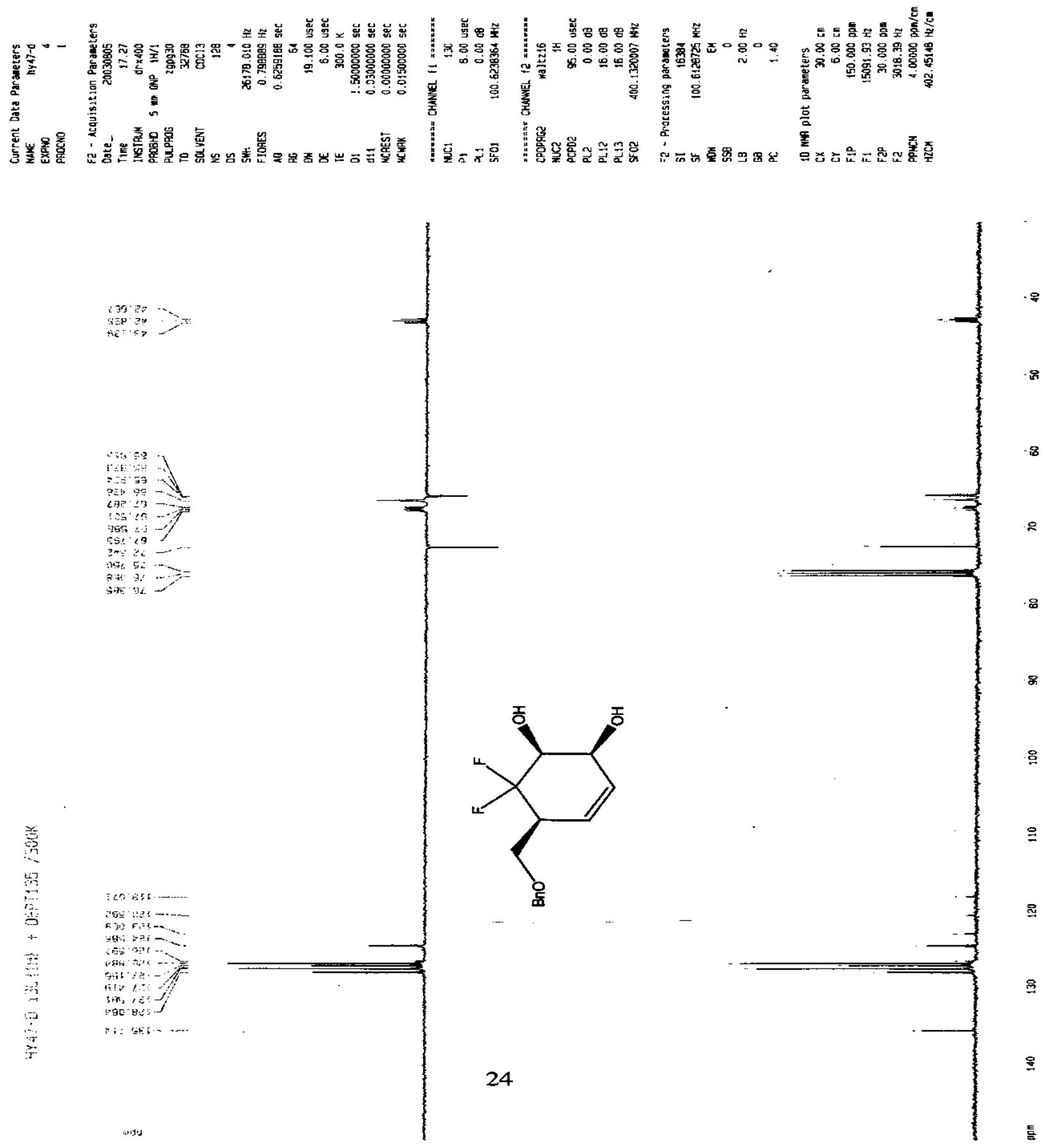


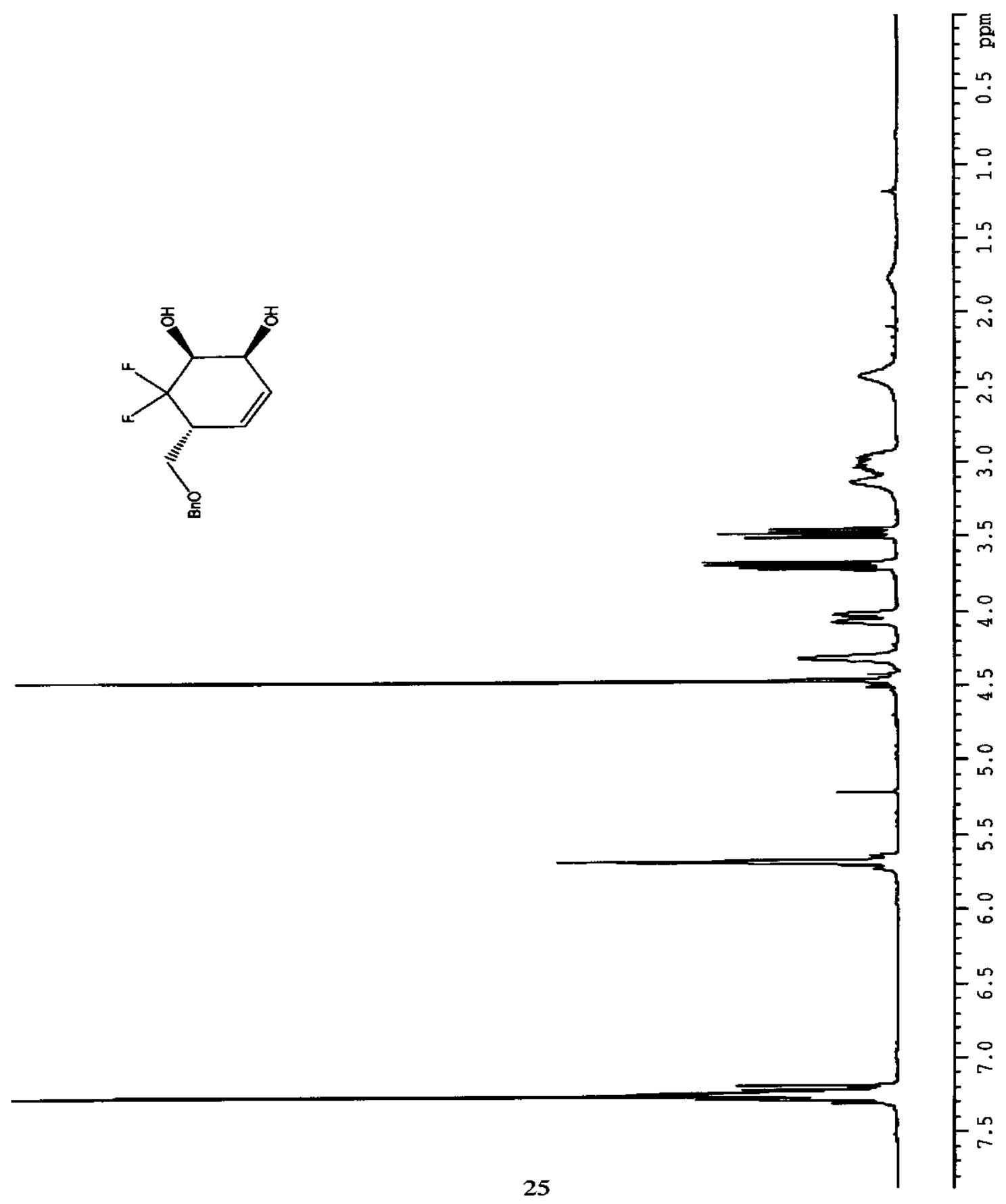




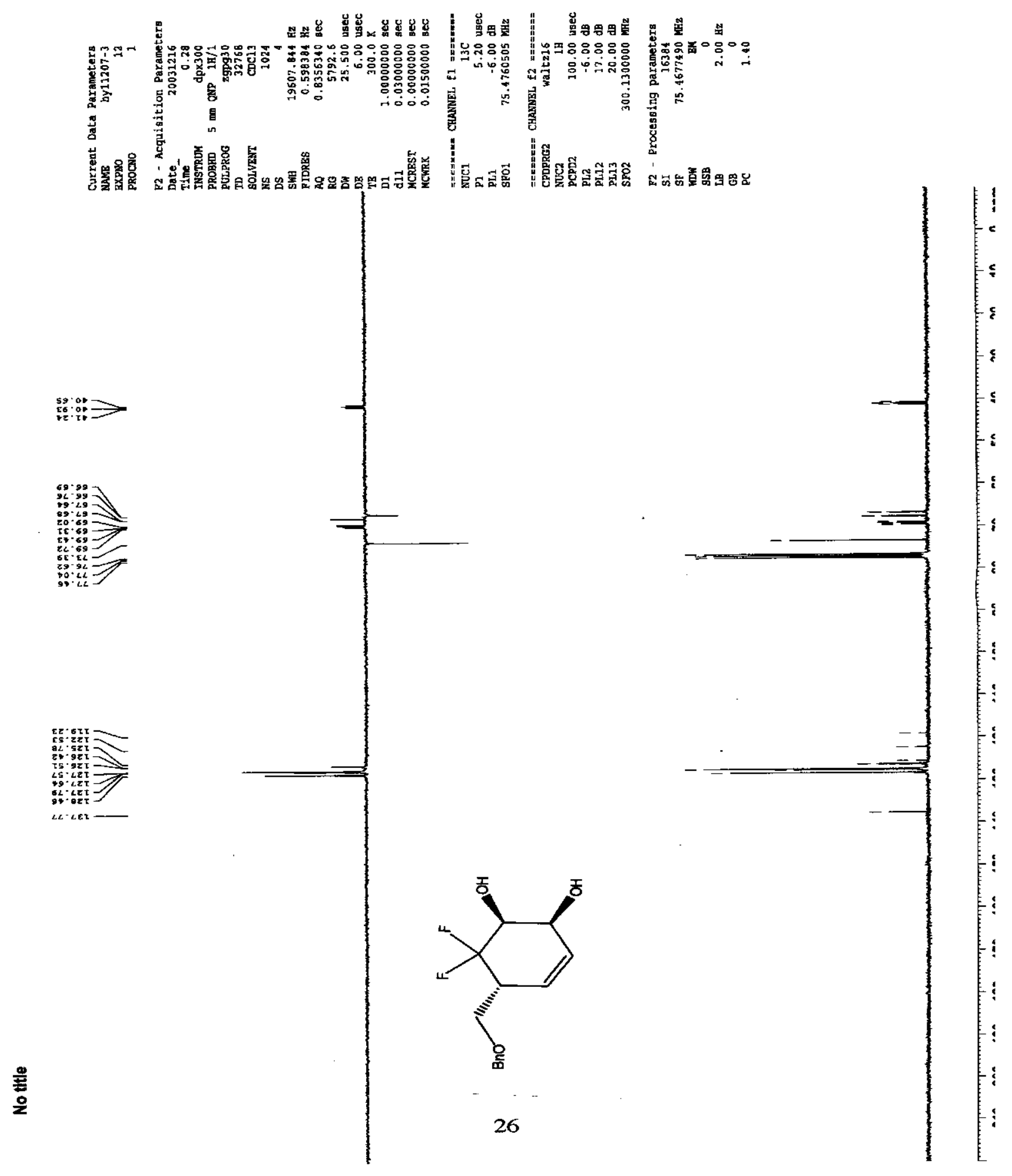




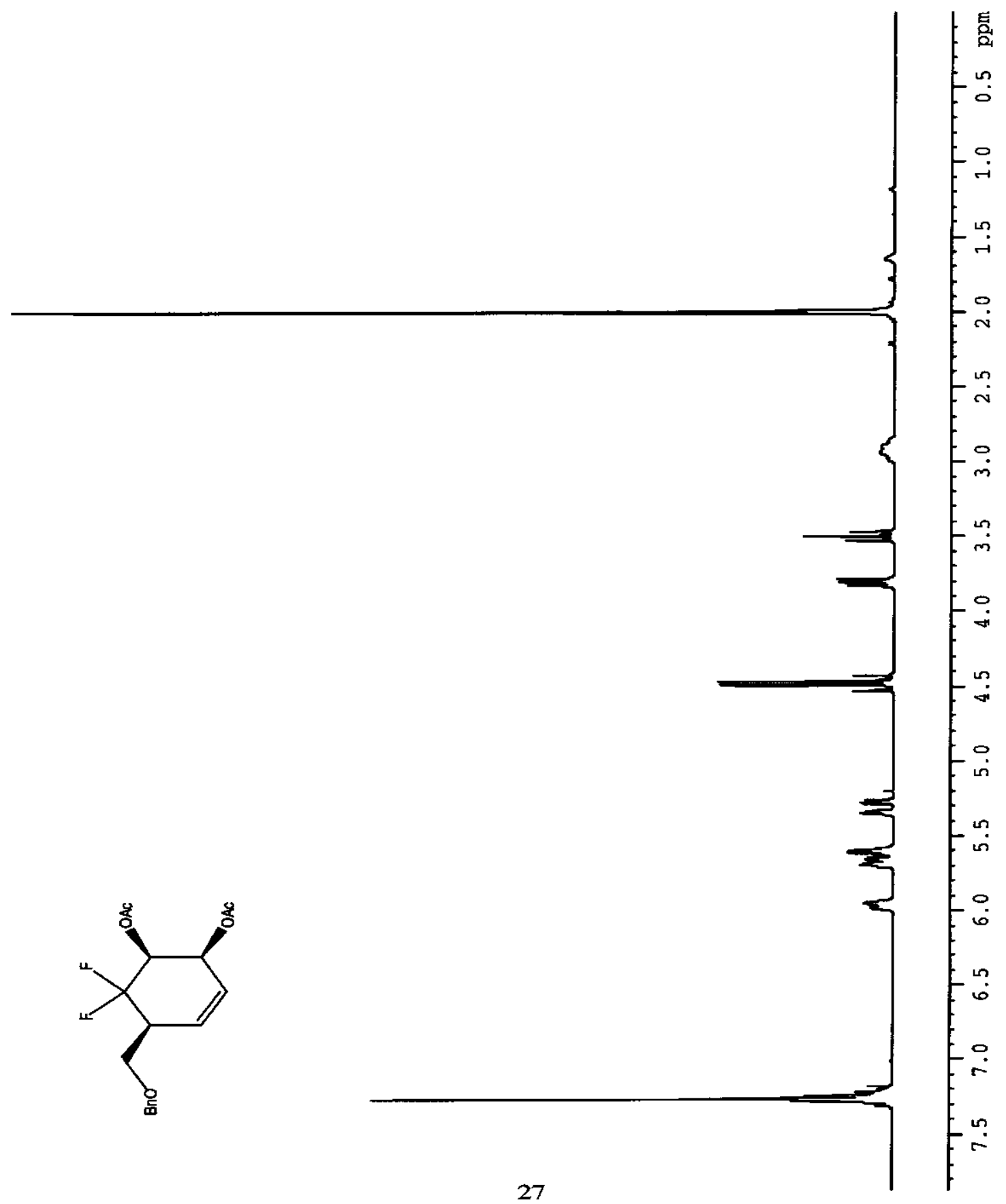




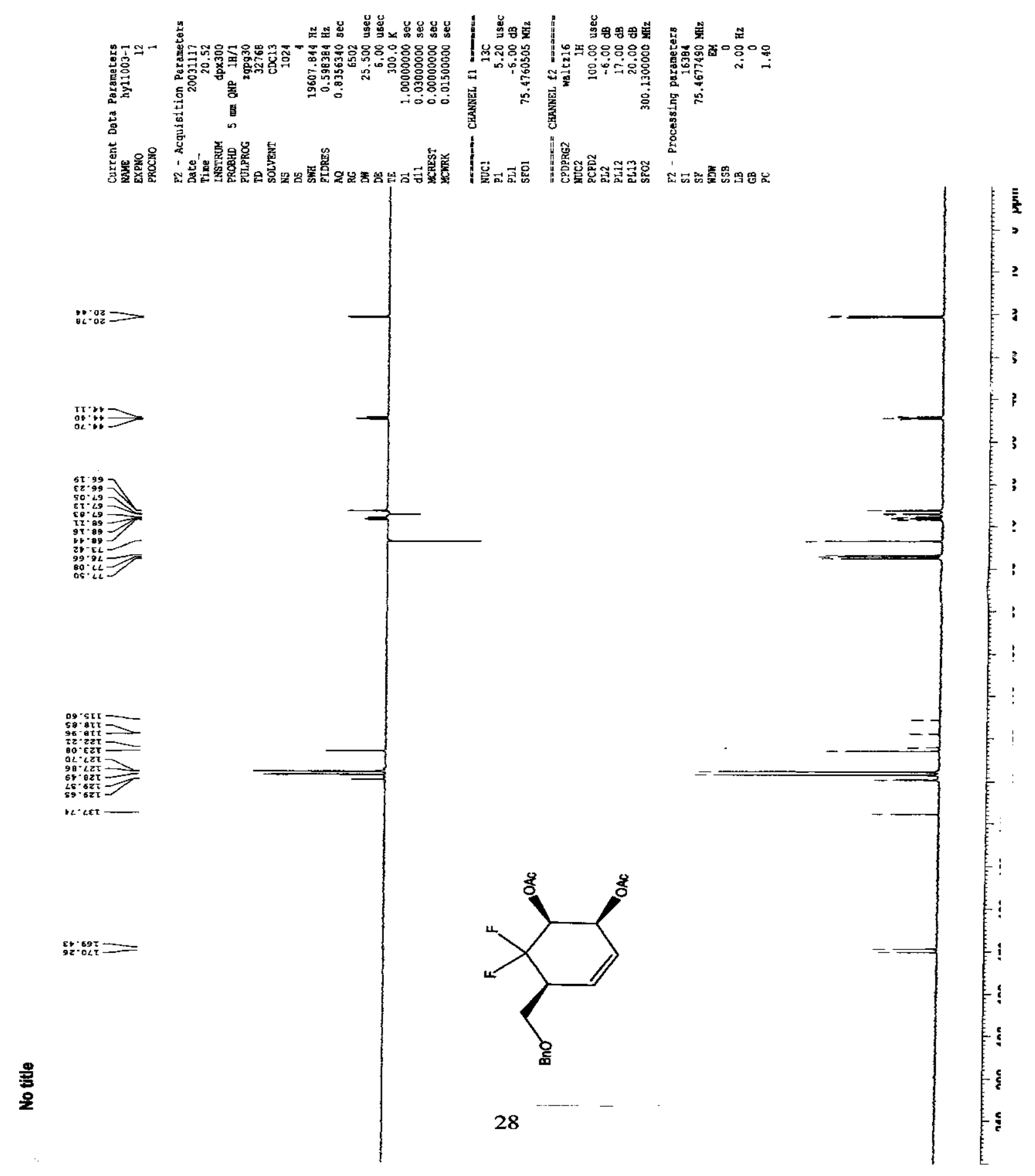




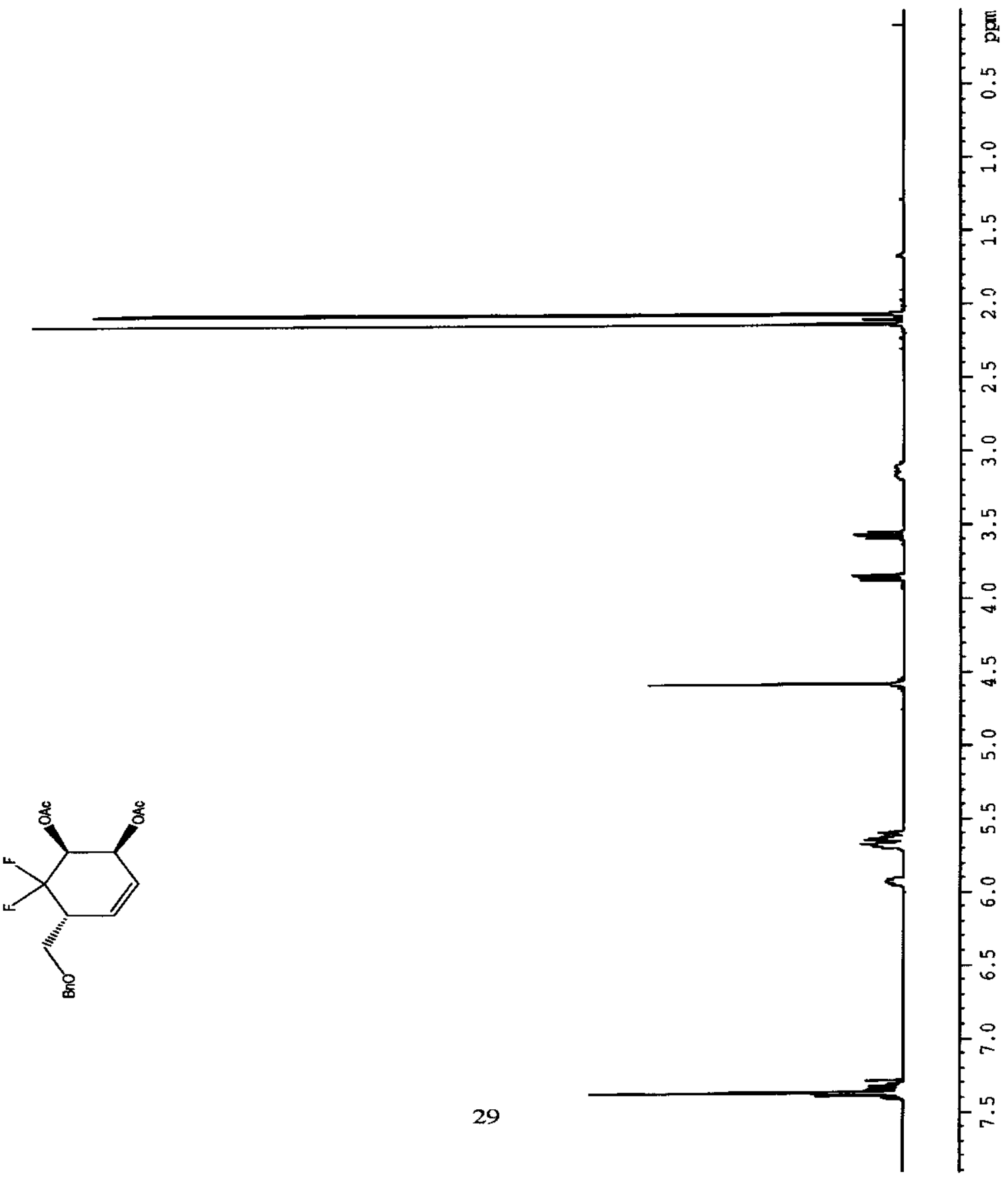



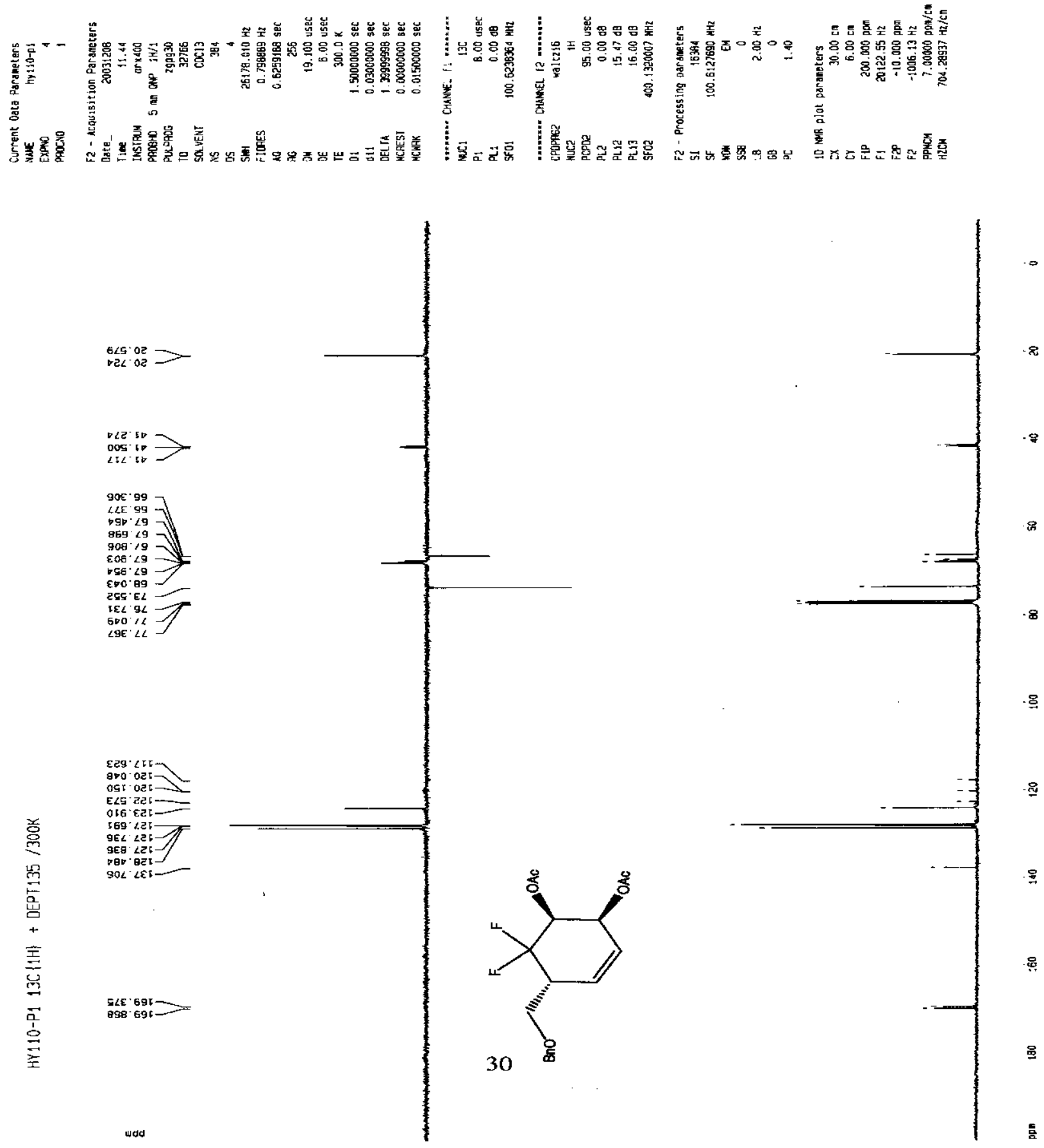


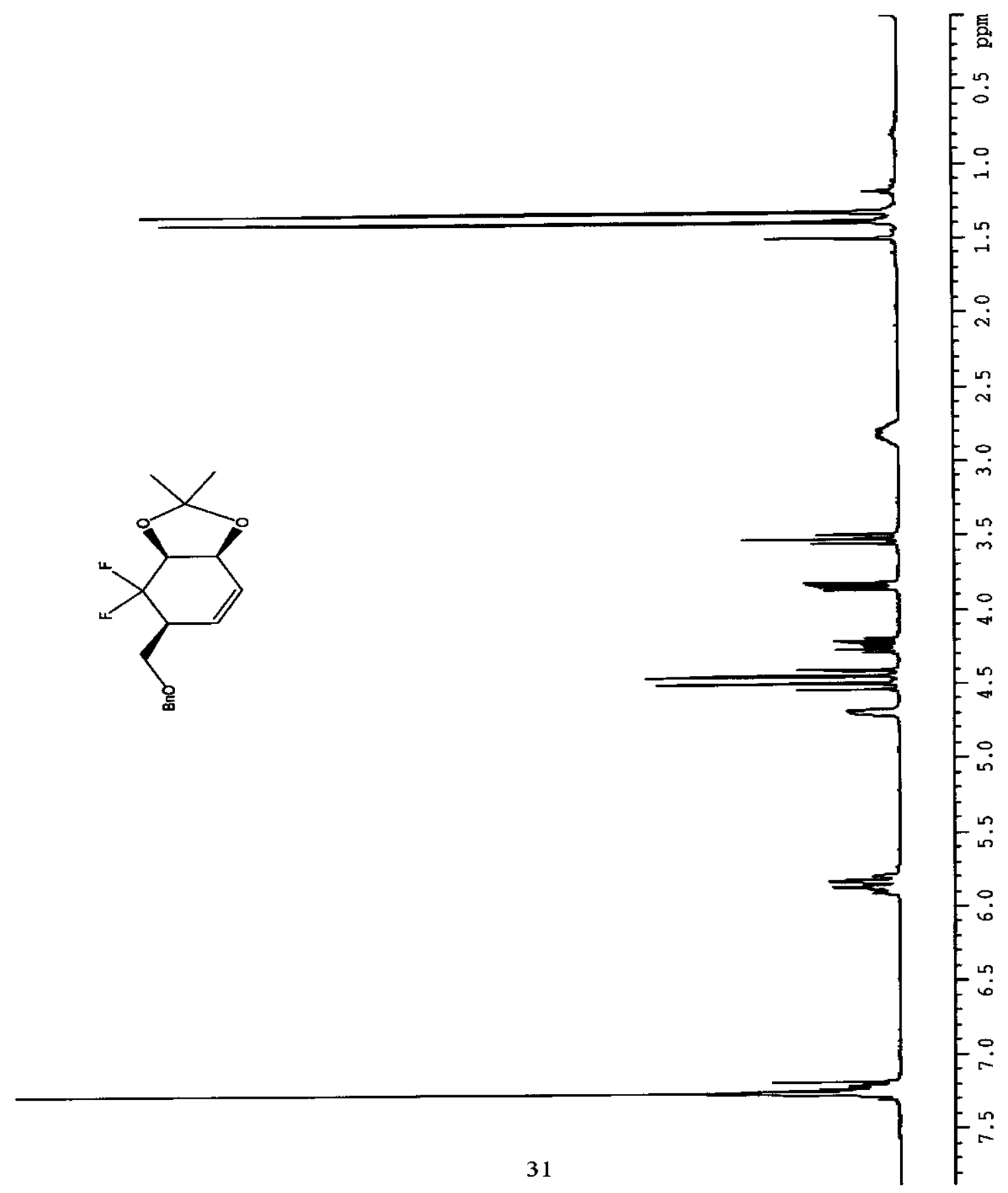




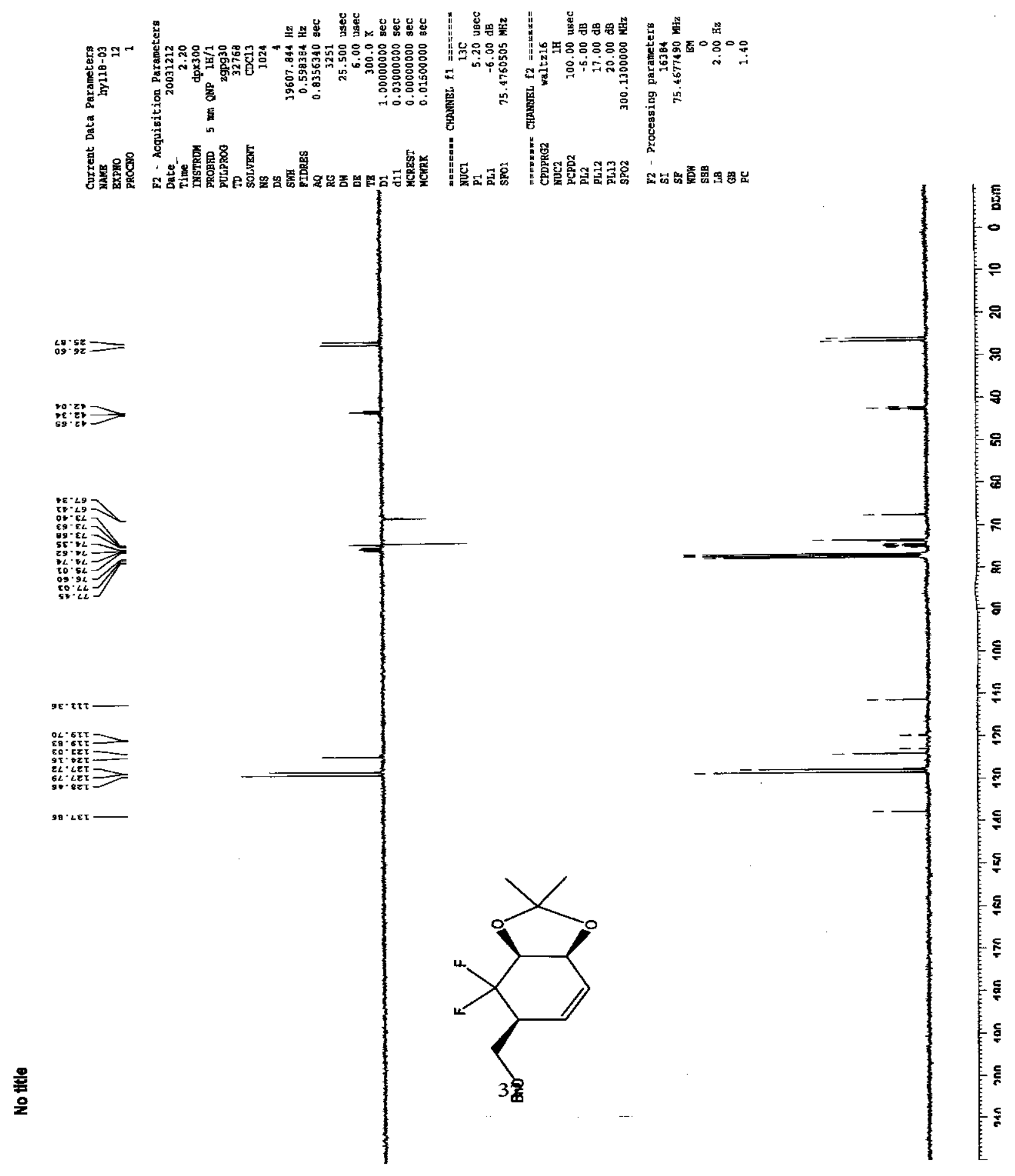




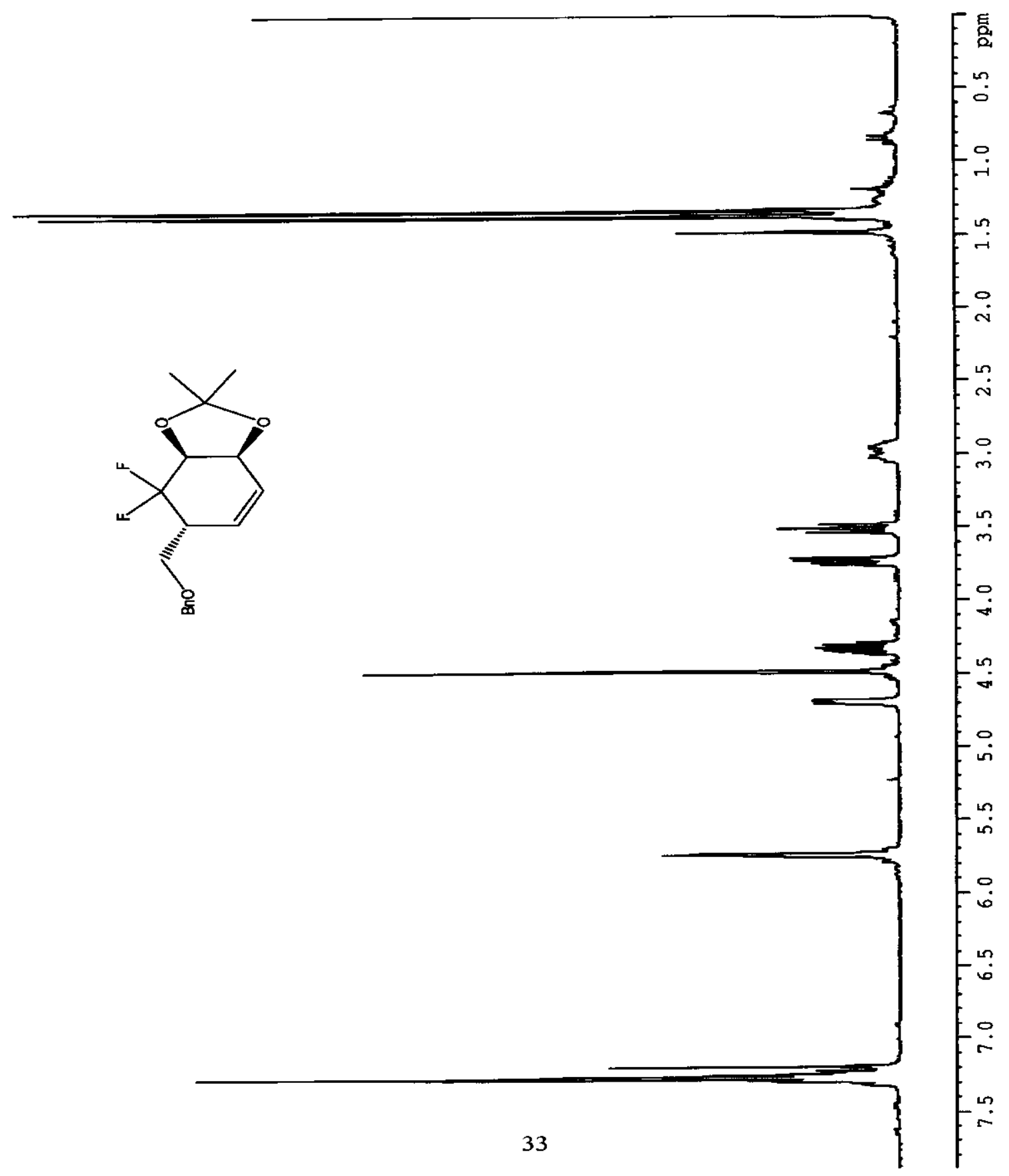




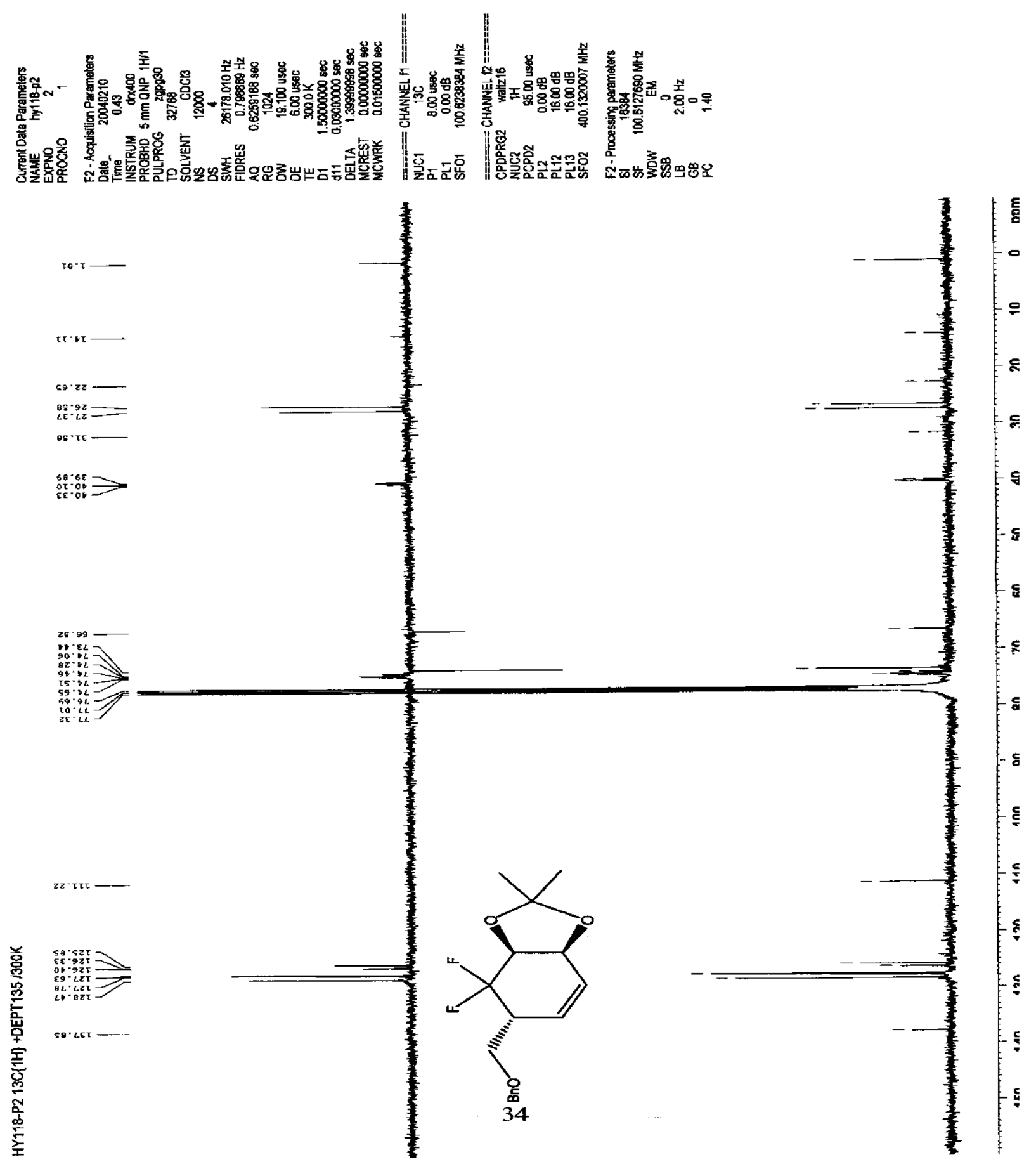



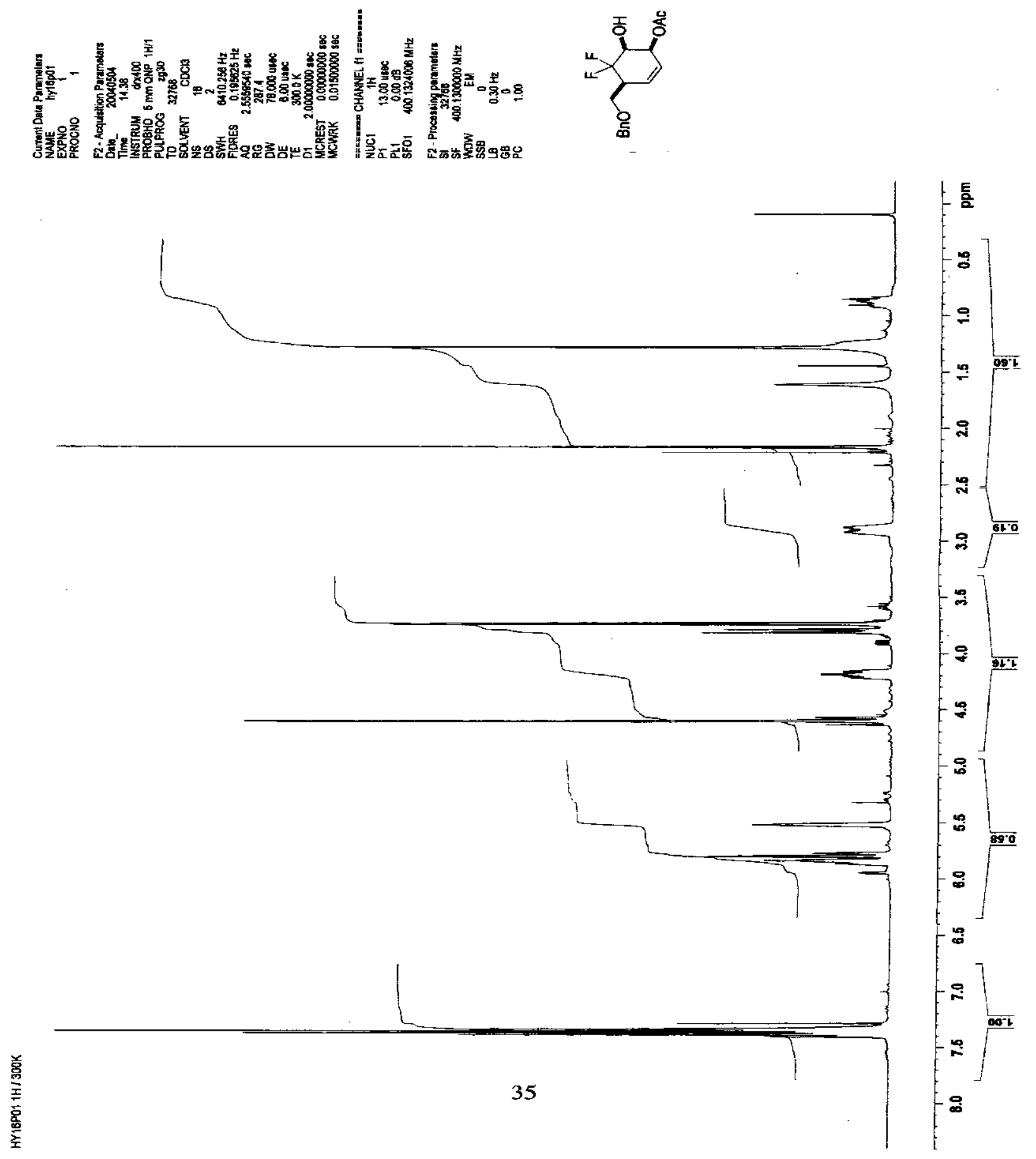


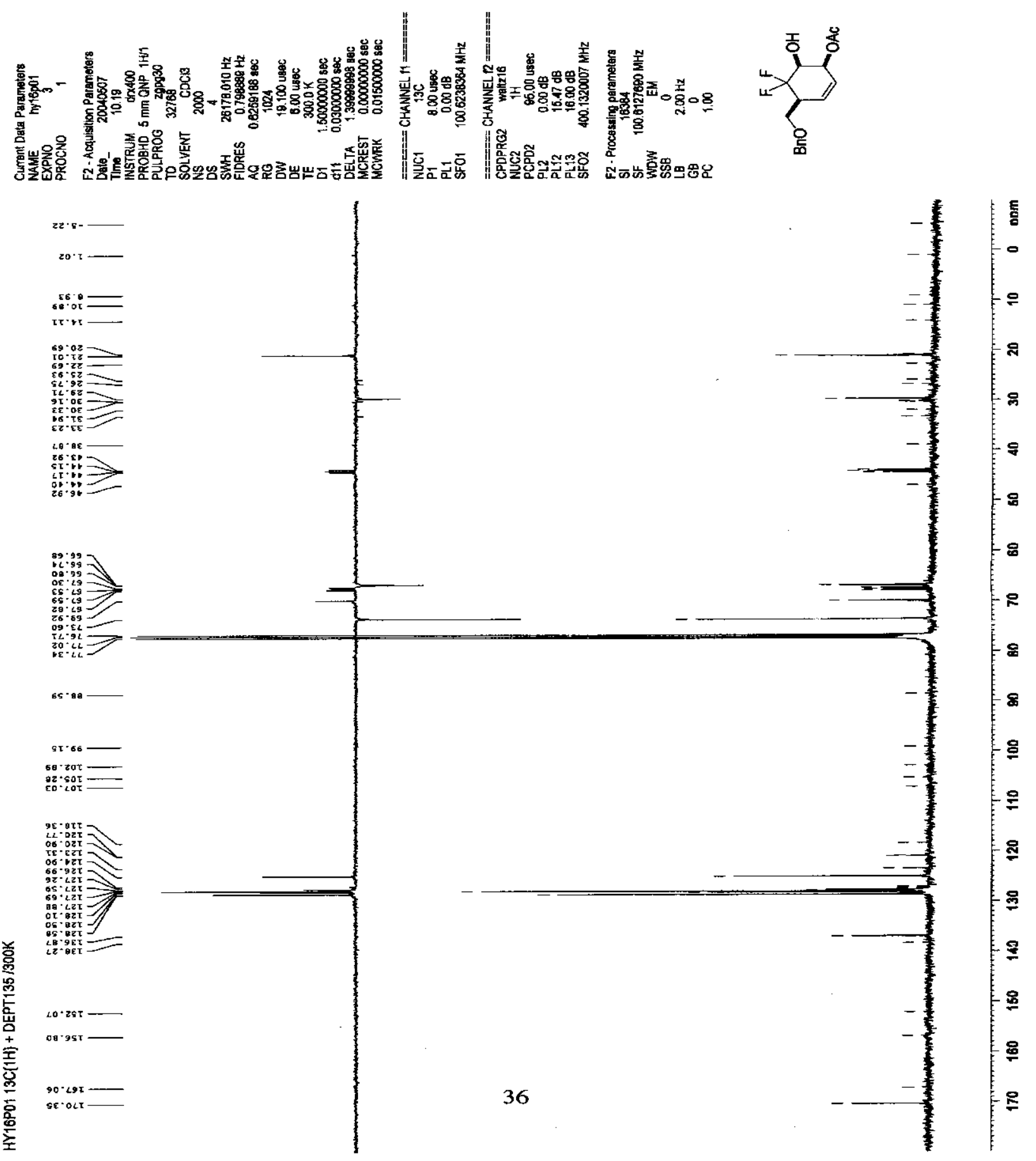



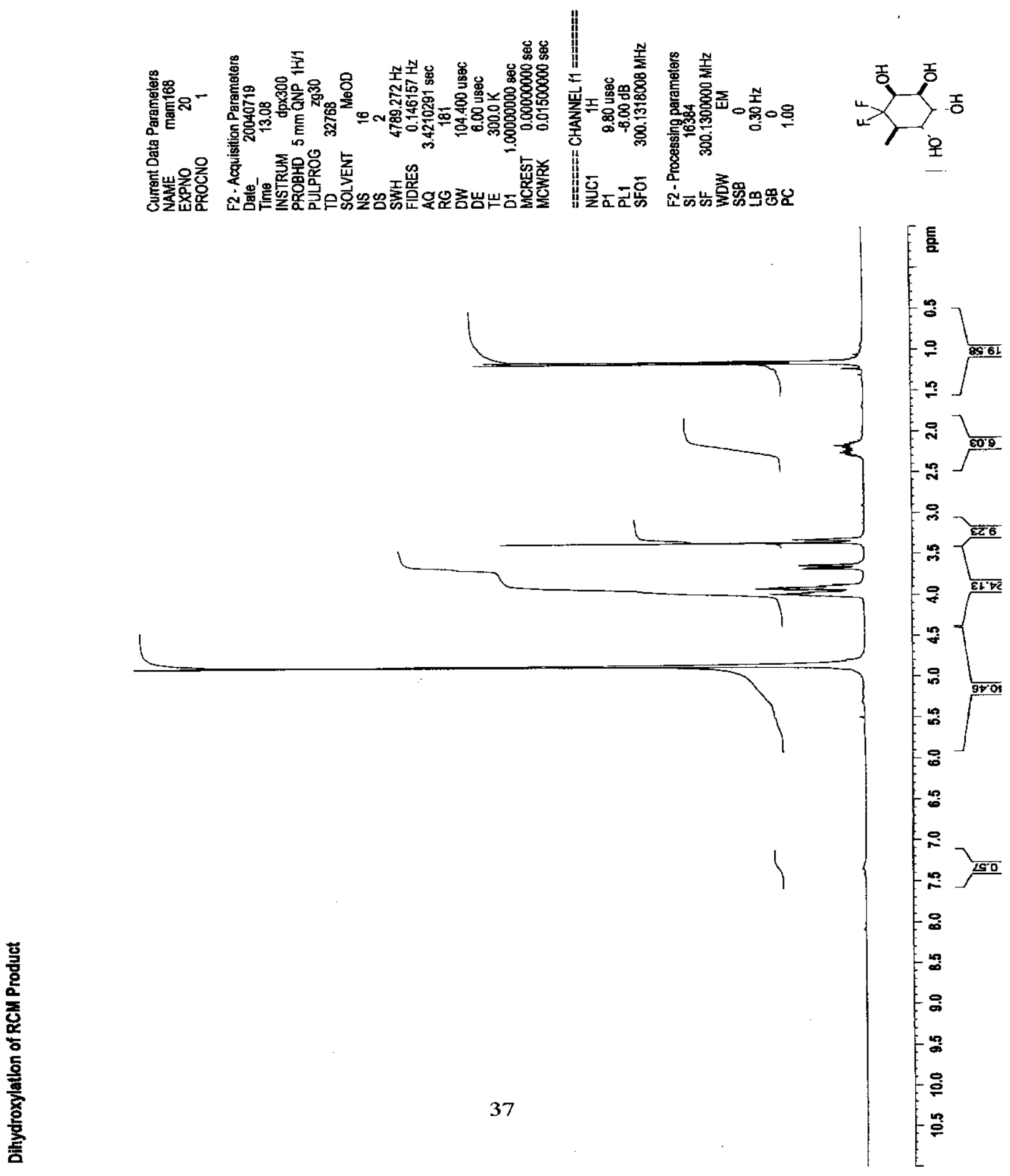


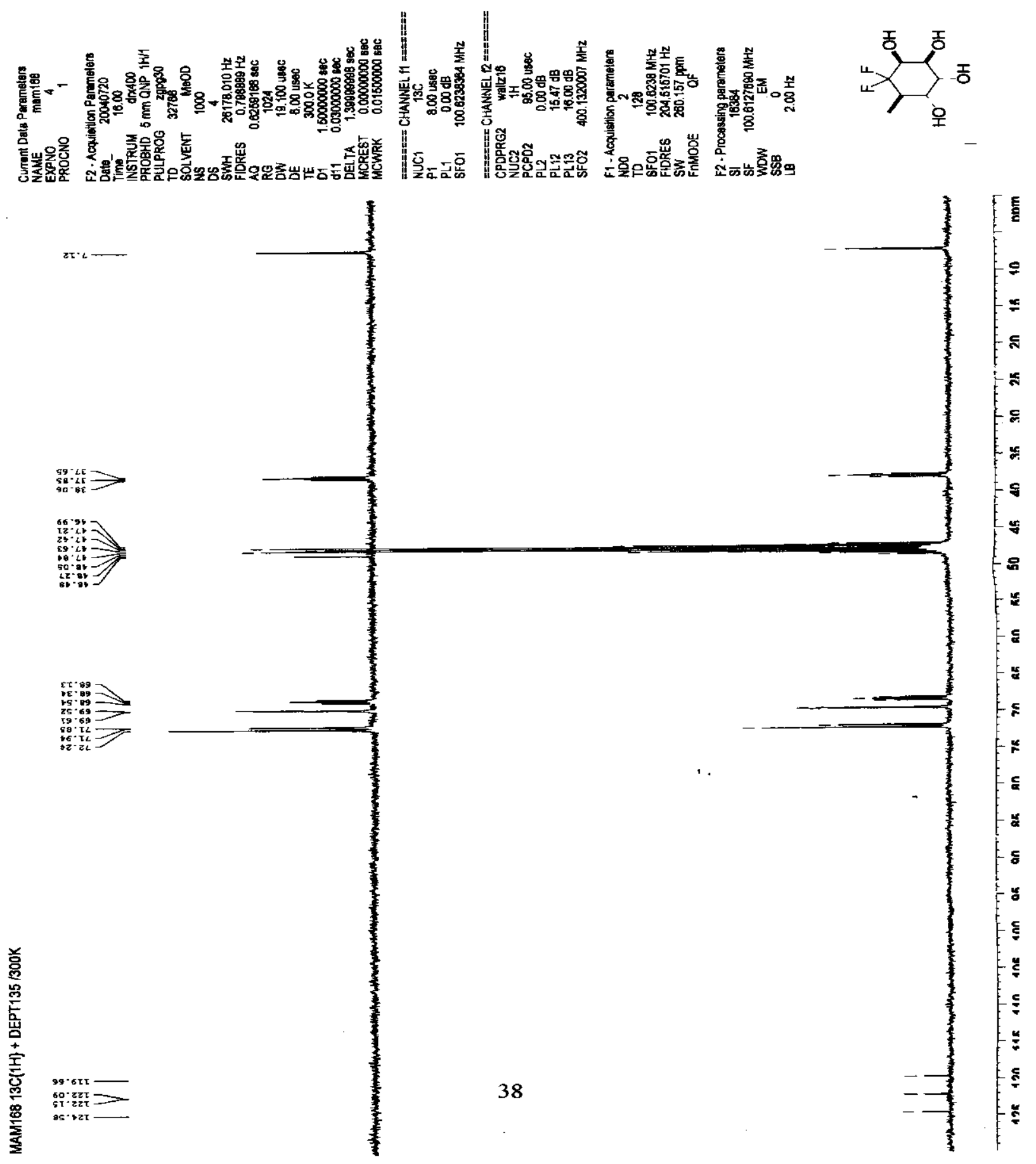

